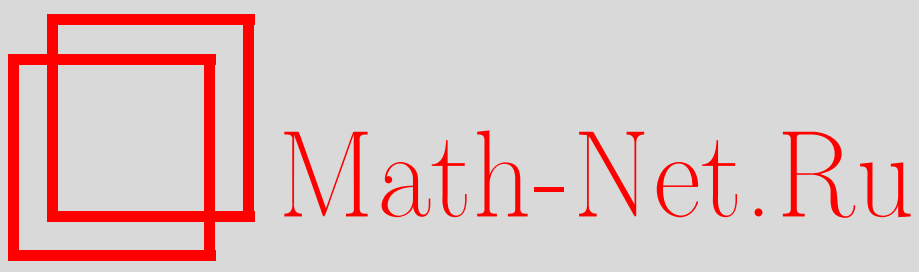

Вик. С. Куликов, О гипотезе Кизини, Изв. РАН. Сер. матем., 1999, том 63, выпуск $6,83-116$

DOI: https://doi.org/10.4213/im267

Использование Общероссийского математического портала Math-Net.Ru подразумевает, что вы прочитали и согласны с пользовательским соглашением

http://www . mathnet.ru/rus/agreement

Параметры загрузки:

IP : 54.174 .149 .18

26 апреля 2023 г., $17: 44: 32$ 
УДК $512.7+515.1$

\author{
Вик. С. Куликов
}

\title{
О гипотезе Кизини
}

Гипотеза Кизини утверждает, что для каспидальной кривой $B \subset \mathbb{P}^{2}$ общий морфизм $f, \operatorname{deg} f \geqslant 5$, гладкой проективной поверхности на $\mathbb{P}^{2}$, разветвленной вдоль $B$, единствен с точностью до изоморфизма. В статье доказано, что если $\operatorname{deg} f$ больше, чем значение некоторой функции, зависящей от степени, рода и числа каспов кривой $B$, то гипотеза Кизини выполнена для $B$. Это неравенство имеет место для почти всех общих морфизмов. В частности, оно выполняется для общих морфизмов поверхностей с обильным каноническим классом, заданных линейной подсистемой $m$-го канонического класса, $m \in \mathbb{N}$.

Кроме того, в статье приведены примеры пар кривых $B_{1, m}, B_{2, m} \subset \mathbb{P}^{2}$ $(m \in \mathbb{N}, m \geqslant 5)$ плоских каспидальных кривых таких, что:

(i) $\operatorname{deg} B_{1, m}=\operatorname{deg} B_{2, m}$ и в $\mathbb{P}^{2}$ можно найти гомеоморфные друг другу трубчатые окрестности этих кривых, но пары $\left(\mathbb{P}^{2}, B_{1, m}\right)$ и $\left(\mathbb{P}^{2}, B_{2, m}\right)$ не гомеоморфны;

(ii) $B_{i, m}$ - дискриминантная кривая общего морфизма $f_{i, m}: S_{i} \rightarrow \mathbb{P}^{2}$, $i=1,2$, поверхности общего типа $S_{i}$;

(iii) $S_{1}$ и $S_{2}$ - гомеоморфные поверхности (рассматриваемые как четырехмерные действительные многообразия);

(iv) морфизм $f_{i, m}$ задается трехмерной линейной подсистемой из $m$-канонического класса поверхности $S_{i}$.

Библиография: 29 наименований.

\section{Введение}

Пусть $B \subset \mathbb{P}^{2}$ - неприводимая каспидальная плоская кривая над $\mathbb{C}$, т.е. кривая, все особые точки которой суть каспы (особые точки типа $A_{2}$ с локальным уравнением $y^{2}=x^{3}$ ) и ноуды (особые точки типа $A_{1}$ с локальным уравнением $y^{2}=x^{2}$ ). Обозначим через $2 d$ степень кривой $B$, и пусть $g$ - род ее десингуляризации, $c-$ число каспов и $n$ - число ноудов. Назовем $B$ дискриминантной кривой общего морфизма, если существует конечный морфизм $f: S \rightarrow \mathbb{P}^{2}, \operatorname{deg} f \geqslant 3$, удовлетворяющий следуюшим условиям:

(i) $S$ - неособая неприводимая проективная поверхность;

(ii) $f$ неразветвлен над $\mathbb{P}^{2} \backslash B$;

(iii) $f^{*}(B)=2 R+C$, где $R$ - неособая неприводимая кривая, а $C$ - приведенная кривая;

(iv) $f_{\mid R}: R \rightarrow B$ совпадает с морфизмом нормализации кривой $B$.

Мы будем называть такой морфизм $f$ общим морфизмом.

Работа выполнена при частичной финансовой поддержке РФФИ (№ 99-01-01133) и INTAS.

(C) Вик.С. Куликов 1999 
Отметим, что если $S \subset \mathbb{P}^{r}$, морфизм $f$ является ограничением на $S$ общей проекции проективного пространства $\mathbb{P}^{r}$ на плоскость $\mathbb{P}^{2}$ и $B$ - кривая ветвления морфизма $f$, то $(S, f)$ - общий морфизм и $B$ - его дискриминантная кривая.

Два общих морфизма $\left(S_{1}, f_{1}\right),\left(S_{2}, f_{2}\right)$ с одной и той же дискриминантной кривой $B$ называются әквивалентными, если существует изоморфизм $\varphi: S_{1} \rightarrow S_{2}$ такой, что $f_{1}=f_{2} \circ \varphi$. Мы будем говорить, что общий морфизм $f$ однозначно определяется своей дискриминантной кривой $B$, если любые два общих морфизма с дискриминантной кривой $B$ эквивалентны.

Следуюшее утверждение известно как гипотеза Кизини.

ГиПоТЕЗА 1. Если $f: S \rightarrow \mathbb{P}^{2}$ - общий морфизм степени $\operatorname{deg} f \geqslant 5$, то $f$ однозначно определяется своей дискриминантной кривой.

Если $B \subset \mathbb{P}^{2}$ - двойственная кривая к гладкой кубике, то $B$ является дискриминантной кривой четырех неэквивалентных общих морфизмов (см. [9], [6]). Три из них имеют степень четыре, а четвертьй имеет степень три. Это - единственный известный к настоящему моменту пример дискриминантной кривой нескольких неэквивалентных общих морфизмов. В п. 1.2 приведен еще ряд примеров кривых, являющихся дискриминантными кривыми неэквивалентных общих морфизмов (степени $\leqslant 4)$.

В общем случае, как это следует из [6], число неэквивалентных общих морфизмов с заданной дискриминантной кривой $B$ не превосходит $2^{2 g+c-1}$.

Гипотеза Кизини была доказана Б. Мойшезоном для дискриминантных кривых общих проекций гладких гиперповерхностей в $\mathbb{P}^{3}$. Его доказательство использует полученное им в [19] представление фундаментальной группы дополнения в $\mathbb{P}^{2}$ к дискриминантной кривой общей проекции. Краткий обзор результатов, относяшихся к гипотезе Кизини, и попыток доказать ее содержится в [6].

Основным результатом данной статьи является следующая теорема.

ТеОРема 1. Пусть $B$ - дискриминантная кривая общего морфизма $f: S \rightarrow \mathbb{P}^{2}$ степени $\operatorname{deg} f=N$. Если

$$
N>\frac{4(3 d+g-1)}{2(3 d+g-1)-c},
$$

то $f$ однозначно определяется своей дискриминантной кривой B, т.е. гипотеза Кизини верна для дискриминантной кривой $B$.

Теорема 1 показывает, что если степень общего морфизма с данной дискриминантной кривой $B$ достаточно велика, то этот общий морфизм единствен для $B$. Почти все общие морфизмы, интересные с алгебро-геометрической точки зрения, удовлетворяют этому условию. Более точно, пусть $E=f^{*}\left(\mathbb{P}^{1}\right)$ - прообраз общей прямой $\mathbb{P}^{1} \subset \mathbb{P}^{2}$. Мы проверим неравенство (1) для общих морфизмов, заданных трехмерными линейными подсистемами различных линейных систем $|E|$ на поверхностях различных типов, чтобы получить следуюшие теоремы в качестве следствий основного результата. 
ТЕОРЕМа 2. Пусть $S$ - произвольная проективная неособая поверхность и $L$ - произвольный обильньй дивизор на $S, f: S \rightarrow \mathbb{P}^{2}-$ общий морфизм, заданньй трехмерной линейной подсистемой $\{E\} \subset|m L|, \quad m \in \mathbb{Q}$, и пусть $B$ - его дискриминантная кривая. Тогда существует такая константа $m_{0}$ (зависящая от $\left.L^{2},\left(K_{S}, L\right), K_{S}^{2}, p_{a}\right)$, что для дискриминантной кривой $B$ морфизма $f$ этот общий морфизм является единственныц, если $m \geqslant m_{0}$. В частности, если $L=K_{S}$, то ми можем взять $m_{0}=2$.

ТЕОРема 3. Общий морфизм $f: S \rightarrow \mathbb{P}^{2}$ поверхности основного типа $S$ с обильным каноническим классом $K_{S}$, заданный трехмерной линейной системой $\{E\}$, где $E \equiv m K_{S}, m \in \mathbb{N}$ (三 означает численную эквивалентность), однозначно определяется своей дискриминантной кривой $B$.

ТеОРема 4. Обций морфизм $f: S \rightarrow \mathbb{P}^{2}$ поверхности дель Пецио $S$, заданный трехмерной линейной системой $\{E\}$, где $E \in\left|-m K_{S}\right|, \quad m \in \mathbb{N}$, однозначно определяется своей дискриминантной кривой $B$.

ТЕОРема 5. Произвольный общий морфизм $f: S \rightarrow \mathbb{P}^{2}$ поверхности $S=$ $\mathbb{P}^{1} \times \mathbb{P}^{1}$ однозначно определяется своей дискриминантной кривой $B$.

ТЕОРема 6. Произвольный общий морфизм $f: S \rightarrow \mathbb{P}^{2}$ K3-поверхности $S$ однозначно определяется своей дискриминантной кривой $B$.

ТЕОрема 7. Произвольный общий морфизм $f: S \rightarrow \mathbb{P}^{2}$ поверхности Энриквеса $S$ однозначно определяется своей дискриминантной кривой $B$, за исключением, возмохсно, случая $\operatorname{deg} f=4$. В исключительном случае $\operatorname{deg} B=12$, $g=19, c=36, n=0$, и если такой общий морфизм существует, то:

1) существуют по крайней мере два общих морфизма с одной и той жее дискриминантной кривой $B$;

2) каждый общий морфизм $f^{\prime}$ с такой дискриминантной кривой $B$ имеет $\operatorname{deg} f^{\prime} \leqslant 4$.

В частности, гипотеза Кизини верна для всех дискриминантных кривых общих морфизмов поверхностей Энриквеса.

ТЕОРема 8. Произвольный общий морфизм $f: S \rightarrow \mathbb{P}^{2}$ абелевой поверхности $S$ однозначно определяется своей дискриминантной кривой $B$, за исключением, возможсно, случая $\operatorname{deg} f=6$. В исключительном случае $\operatorname{deg} B=18$, $g=28, c=72, n=36$, и если для $B$ существует общий, неэквивалентный $f$, морфизм $f^{\prime}$, mо $\operatorname{deg} f^{\prime} \leqslant 6$.

Теорема 9. Общий морфизм $f: S \rightarrow \mathbb{P}^{2}$ полного пересечения $S \subset \mathbb{P}^{k+2}$, индуцированный проекцией $\mathrm{pr}: \mathbb{P}^{k+2} \rightarrow \mathbb{P}^{2}$, однозначно определяется своей дискриминантной кривой $B$.

Теорема 10. Гипотеза Кизини выполнена для дискриминантных кривых $B$, являющихся двойственнымми кривыми к плоским нодальньмм кривым (т.е. к кривым с лишь ноудами в качестве особых точек), за исключением, возможнно, следующих случаев:

1) $\operatorname{deg} B=30, \quad g=10, \quad c=72, \quad n=324$;

2) $\operatorname{deg} B=20, \quad g=6, \quad c=45, \quad n=120$; 
3) $\operatorname{deg} B=18, \quad g=5, \quad c=39, \quad n=92$;

4) $\operatorname{deg} B=16, g=4, c=33, n=68$.

Во всех исключительных случаях, если существуют неэквивалентные общие морфизмы с данной дискриминантной кривой $B$, эти морфизмы имеют степень $\leqslant 6$.

Tеорема 11. Гипотеза Кизини выполнена для дискриминантных кривых $B$ рода $g \leqslant 3$.

Теорема 12. Гипотеза Кизини верна для дискриминантных кривых $B$, удовлетворяющих неравенству $d>3(g-1)$.

K сожалению, в общем случае мы не имеем удовлетворительного описания (в алгебро-геометрических терминах) множества дискриминантных кривых с заданными степенью, родом и числом каспов. С другой стороны, множество дискриминантных кривых может быть полностью описано в терминах фундаментальной группы дополнения к ним в $\mathbb{P}^{2}$ (см. предложение 1 в п. 1.2). Однако алгебро-геометрическое описание множества дискриминантных кривых может быть дано в некоторых частных случаях. Например, в [29] Зариский доказал, что секстика с шестью каспами является дискриминантной кривой обшего морфизма тогда и только тогда, когда эти 6 особых точек лежат на конике, и он доказал сушествование секстик с шестью каспами, не лежащими на одной и той же конике. В этой статье мы также дадим описание в терминах алгебраической геометрии множества дискриминантных кривых морфизмов, заданных трехмерными подсистемами $m$-го канонического класса для $m \geqslant 21$ (см. п. 4.2). Такие дискриминантные кривые мы будем называть кривыми .

Две плоские кривые $B_{1}, B_{2} \subset \mathbb{P}^{2}, \operatorname{deg} B_{1}=\operatorname{deg} B_{2}$, над $\mathbb{C}$ назьваются парой Зариского, если в $\mathbb{P}^{2}$ можно найти гомеоморфные друг другу трубчатые окрестности этих кривых, но пары $\left(\mathbb{P}^{2}, B_{1}\right)$ и $\left(\mathbb{P}^{2}, B_{2}\right)$ не гомеоморфны.

Первый пример такой пары был построен Зариским: это как раз упомянутые выше две плоские кривые $B_{1}$ и $B_{2}$ степени 6 с шестью обыкновенными каспами. В данной статье будет доказано сушествование большого числа различных пар Зариского, являющихся дискриминантными кривыми общих морфизмов на $\mathbb{P}^{2}$.

Пусть $\left(\mathbb{P}^{2}, B_{1}\right)$ и $\left(\mathbb{P}^{2}, B_{2}\right)$ - две гомеоморфные пары. Из предложения 1 следует, что если $B_{1}$ является дискриминантной кривой обшего морфизма $\left(S_{1}, f_{1}\right)$, то $B_{2}$ также является дискриминантной кривой некоторого обшего морфизма $\left(S_{2}, f_{2}\right)$. Более того, если морфизм $\left(S_{1}, f_{1}\right)$ однозначно определяется своей дискриминантной кривой $B_{1} \subset \mathbb{P}^{2}$, то то же самое верно и для морфизма $\left(S_{2}, f_{2}\right)$. Кроме того, в этом случае (см. предложение 8 в $\S 5$ ) $S_{1}$ и $S_{2}$ гомеоморфны друг другу. Отсюда естественньм образом возникает следующий вопрос.

ПроБлема 1. Пусть $S_{1}, S_{2} \subset \mathbb{P}^{r}-$ две гомеоморфные (случай С) (соответственно диффеоморфные (случай D)) поверхности общего типа, вложенные с помощью т-канонического класса в проективное пространство, и пусть $B_{i}-$ m-канонические дискриминантные кривые общих проекций поверхностей $S_{i}$ на $\mathbb{P}^{2}$. Будут ли пары $\left(\mathbb{P}^{2}, B_{1}\right)$ и $\left(\mathbb{P}^{2}, B_{2}\right)$ гомеоморфны (соответственно диффеоморфны)? 
В случае $\mathrm{C}$ данная проблема имеет отрицательное решение, а именно имеет место следуюшая теорема.

ТЕОРЕМа 13. Для каждого натурального числа $k$ существует набор $\left(B_{1, m}, \ldots, B_{k, m}\right), \quad m \in \mathbb{N}, \quad m \geqslant 5$, состоящий из $k m$-канонических кривых $B_{i, m}$ таких, что пары $B_{i, m}, B_{j, m}$ при $i \neq j$ являются парами Зариского, но соответствующие этим кривым общие морфизмы $f_{i, m}: S_{i} \rightarrow \mathbb{P}^{2}$, $i=1, \ldots, k$, являются морфизмами попарно гомеоморфных (но недиффеоморфных) поверхностей общего типа $S_{i}$.

На самом деле будет доказано несколько более сильное, чем теорема 13, утверждение. А именно, будет доказано, что гладкие структуры, возникающие из комплексных структур на гомеоморфных поверхностях $S_{1}$ и $S_{2}$ общего типа (рассматриваемых как четырехмерные действительные многообразия) и составляющих пару Катанезе (определение пары Катанезе см. в § 6), различаются топологчческими типами пар $\left(\mathbb{P}^{2}, B_{1, m}\right)$ u $\left(\mathbb{P}^{2}, B_{2, m}\right)$, где $B_{i, m}-$ т-канонические дискриминантные кривые общих морфизмов $S_{i}$ на $\mathbb{P}^{2}$, заданных т-каноническим классом $\left|m K_{S_{i}}\right|$.

Это позволяет надеяться на то, что и в общем случае гладкие структуры на гомеоморфных проективных поверхностях общего типа различаются с помощью топологических типов вложений в $\mathbb{P}^{2}$ соответствующих этим поверхностям $m$-канонических дискриминантных кривых.

В случае D проблема 1 к настоящему моменту остается открытой.

В $\S 1$ мы напомним хорошо известные факты об общих морфизмах и их дискриминантных кривых, $\S 2$ посвящен доказательству теоремы 1. В $\S 3$ мы проверим неравенство (1) в различных случаях, чтобы доказать теоремы $2-12 ; \S 4$ содержит более детальное исследование случая, когда общий морфизм задан линейной подсистемой $m$-го канонического класса. В $\S 5$ кратко обсуждается вопрос о числе неприводимых компонент пространства модулей дискриминантных кривых с фиксированными степенью, родом и числом каспов. Кроме того, мы применяем теорему 3 для нахождения новых примеров пар Зариского; $\S 6$ посвящен доказательству теоремы 13.

Данная статья была написана во время пребывания автора в Институте математики Макса Планка (Бонн, ФРГ).

\section{$\S 1$. Общие морфизмы и их дискриминантные кривые}

1.1. Пусть $B$ - дискриминантная кривая общего морфизма $f: S \rightarrow \mathbb{P}^{2}, \operatorname{deg} f=N$, $E=f^{*}\left(\mathbb{P}^{1}\right)$. Имеем $\left(E^{2}\right)_{S}=N$.

Лемма 1. Степень $\operatorname{deg} B=2 d$ является четным числом, в частности $d \in \mathbb{N}$.

ДокАЗАТЕЛЬСТво. По формуле Гурвища $2 g(E)-2=-2 N+\operatorname{deg} B$. Поэтому $\operatorname{deg} B$ - четное число.

Так как $g(E) \geqslant 0$, то мы имеем следуюшее неравенство:

$$
\operatorname{deg} f \leqslant d+1 .
$$


Напомним некоторые неравенства, связываюшие род, степень и число каспов кривой $B$ и вытекающие из формул Плюккера и результата Нори [21]. Пусть $B^{*}-$ кривая, двойственная к $B$. Положим $\delta=\operatorname{deg} B^{*}, \gamma-$ число каспов кривой $B^{*}$ и $\nu$ - число ее ноудов. Из формул Плюккера

$$
\begin{aligned}
\delta & =2 d(2 d-1)-2 n-3 c \\
2 d & =\delta(\delta-1)-2 \nu-3 \gamma \\
2 g & =(2 d-1)(2 d-2)-2 n-2 c \\
2 g & =(\delta-1)(\delta-2)-2 \nu-2 \gamma
\end{aligned}
$$

следует, что

$$
\delta=4 d-c+2 g-2, \quad \gamma=6 d-2 c+6 g-6 .
$$

Так как $\delta \geqslant 0$ и $\gamma \geqslant 0$, то имеет место следующая лемма.

ЛЕмма 2. Имеют место следующие неравенства:

$$
c \leqslant 4 d+2 g-2, \quad c \leqslant 3 d+3 g-3 .
$$

СЛЕДСТВИЕ $1 . c<2(3 d+g-1)$.

ЛЕмМА $3.3 d+g-1 \leqslant 2 c$.

ДокАЗАТЕльСтво. Если $B$ - дискриминантная кривая общего морфизма, то $\pi_{1}\left(\mathbb{P}^{2} \backslash B\right)$ не может быть абелевой группой. Поэтому из [21] следует, что $B^{2}-6 c \leqslant 2 n$. Для плоской каспидальной кривой $B$ степени $\operatorname{deg} B=2 d$ имеем $g+c+n=(2 d-1)(d-1)$. Следовательно, $B^{2}-2 n=4 d^{2}-2 n=2 c+6 d+2 g-2 \leqslant 6 c$.

Лемма 4. Имеют место следующие равенства:

$$
\begin{aligned}
R^{2} & =2 d^{2}-c-n \\
& =3 d+g-1 .
\end{aligned}
$$

ДокаЗАТЕЛЬСТво. Имеем $K_{S}=-3 E+R$. Поэтому (4) следует из формулы присоединения:

$$
\frac{\left(K_{S}+R, R\right)}{2}=\frac{(-3 E+2 R, R)}{2}=-3 d+R^{2}=g-1
$$

Мы получим (3), если вместо $g$ подставим $g=(2 d-1)(d-1)-c-n$.

ЛЕмма 5. Имеет место неравенство

$$
N \leqslant \frac{4 d^{2}}{3 d+g-1},
$$

$u$ (5) является равенством тогда и только тогда, когда либо $E \equiv m K_{S}$ для некоторого $m \in \mathbb{Q}^{*}$, либо $K_{S} \equiv 0$. 
ДокАЗАТЕЛьСтво. По теореме Ходжа об индексе

$$
\left|\begin{array}{cc}
E^{2} & (E, R) \\
(E, R) & R^{2}
\end{array}\right|=\left|\begin{array}{cc}
N & 2 d \\
2 d & 3 d+g-1
\end{array}\right| \leqslant 0
$$

т.е. $N \leqslant 4 d^{2} /(3 d+g-1)$, и мы имеем равенство тогда и только тогда, когда $E$ и $R$ линейно зависимы в $\mathrm{NS}(S) \otimes \mathbb{Q}$, где $\mathrm{NS}(S)$ - группа Нерона-Севери поверхности $S$. В последнем случае так как $K_{S}=-3 E+R$, то либо $E \equiv m K_{S}$ для некоторого $m$, либо $K_{S} \equiv 0$.

ЛЕмма 6. Имеем

$$
\begin{aligned}
K_{S}^{2} & =9 N+2 d^{2}-12 d-c-n \\
& =9 N-9 d+g-1
\end{aligned}
$$

ДокаЗАТЕльство. $K_{S}^{2}=(-3 E+R,-3 E+R)=9 E^{2}-6(E, R)+R^{2}=9 N-$ $12 d+R^{2}$.

Лемма 7. Топологическая Эйлерова характеристика равна:

$$
\begin{aligned}
e(S) & =3 N+4 d^{2}-6 d-3 c-2 n \\
& =3 N+2(g-1)-c
\end{aligned}
$$

ДокАЗАтЕЛЬСтво. Рассматривая общий пучок прямых на $\mathbb{P}^{2}$ и его прообраз $\left\{E_{t}\right\}$ на $S$, мы получим формулу для $e(S)$ :

$$
\begin{aligned}
e(S)+N & =\delta+2(2-2 g(E))=2 d(2 d-1)-3 c-2 n-2\left(K_{S}+E, E\right) \\
& =2 d(2 d-1)-3 c-2 n-2(-2 E+R, E)=4 N+4 d^{2}-6 d-3 c-2 n
\end{aligned}
$$

Из формулы Нётера $K_{S}^{2}+e(S)=12 p_{a}$ следует

ЛЕмма 8. Эйлерова характеристика структурного пучка $\mathscr{O}_{S}$ равна:

$$
\begin{aligned}
p_{a}=1-q+p_{g} & =N+\frac{d(d-3)}{2}-\frac{c}{3}-\frac{n}{4} \\
& =N+\frac{3 g-3-9 d-c}{12} .
\end{aligned}
$$

СЛЕДСТВИЕ 2 [20]. Имеем

$$
c \equiv 0 \quad(\bmod 3), \quad n \equiv 0 \quad(\bmod 4)
$$

ЛЕмма 9. Дивизор $R$ обилен на $S$. 
ДокАЗАТЕльство. Для доказательства достаточно показать, что $(R, \Gamma)>0$ для любой неприводимой кривой $\Gamma$. Если $(R, \Gamma) \leqslant 0$, то по теореме Ходжа об индексе $\Gamma^{2}<0$ и $(R, \Gamma)=0$, так как по лемме 4 индекс самопересечения $R^{2}>0$ и кривая $R$ неприводима. Если $\Gamma^{2}<0$ и $\Gamma$-неприводимая кривая, то $\left(K_{S}, \Gamma\right) \geqslant-1$, т.е. $-3(E, \Gamma)+(R, \Gamma) \geqslant-1$, что противоречит равенству $(R, \Gamma)=0$.

1.2. Зафиксируем какую-нибудь точку $p \in \mathbb{P}^{2} \backslash B$ и обозначим через $\pi_{1}=$ $\pi_{1}\left(\mathbb{P}^{2} \backslash B, p\right)$ фундаментальную группу дополнения к кривой $B$. Выберем произвольную точку $x \in B \backslash \operatorname{Sing} B$ и рассмотрим прямую $\Pi=\mathbb{P}^{1} \subset \mathbb{P}^{2}$, пересекающую $B$ трансверсально в точке $x$. Пусть $\gamma \subset \Pi$ - окружность малого радиуса с центром в $x$. Выбор ориентации на $\mathbb{P}^{2}$ определяет ориентацию на $\gamma$. Пусть $\Gamma-$ петля, состоящая из пути $L$ в $\mathbb{P}^{2} \backslash B$, соединяющего точку $p$ с некоторой точкой $q \in \gamma$, из обхода вдоль $\gamma$ в положительном направлении с началом и конщом в $q$ и возврата в $p$ вдоль $L$ в обратном направлении. Такие петли $\Gamma$ (и соответствующие элементы в $\pi_{1}$ ) мы будем назьвать геометрическими образующими. Хорошо известно, что $\pi_{1}$ порождается геометрическими образующими и что если $B$ - неприводимая кривая, то любые две геометрические образующие сопряжены в $\pi_{1}$.

Для каждой особой точки $s_{i}$ кривой $B$ выберем окрестность $U_{i} \subset \mathbb{P}^{2}$ такую, что $B \cap U_{i}$ в этой окрестности задается (в локальных координатах в $U_{i}$ ) уравнением $y^{2}=x^{3}$, если $s_{i}-$ касп, и $y^{2}=x^{2}$, если $s_{i}$ - ноуд. Пусть $p_{i}$ - произвольная точка в $U_{i} \backslash B$. Хорошо известно, что если $s_{i}-$ касп, то $\pi_{1}\left(U_{i} \backslash B, p_{i}\right)$ изоморфна группе кос $\mathrm{Br}_{3}$ из трех нитей и порождена двумя геометрическими образующими (скажем, $a$ и $b$ ), которые удовлетворяют следующему соотношению:

$$
a b a=b a b .
$$

Если $s_{i}$ - ноуд, то $\pi_{1}\left(U_{i} \backslash B, p_{i}\right)$ изоморфна группе $\mathbb{Z} \oplus \mathbb{Z}$, порожденной двумя коммутируюшими геометрическими образуюшими.

Выберем гладкие пути $\gamma_{i}$ в $\mathbb{P}^{2} \backslash B$, соединяющие точки $p_{i}$ с точкой $p$. Этот выбор определяет гомоморфизмы $\psi_{i}: \pi_{1}\left(U_{i} \backslash B, p_{i}\right) \rightarrow \pi_{1}$. Обозначим образ $\psi_{i}\left(\pi_{1}\left(U_{i} \backslash B, p_{i}\right)\right)$ через $G_{i}$, если $s_{i}-$ касп, и через $\Gamma_{i}$, если $s_{i}-$ ноуд.

Обший морфизм степени $N$ определяет гомоморфизм $\varphi: \pi_{1} \rightarrow \mathfrak{S}_{N}$, где $\mathfrak{S}_{N}-$ симметрическая группа. Этот гомоморфизм $\varphi$ определен однозначно с точностью до внутреннего автоморфизма группы $\mathfrak{S}_{N}$.

ПрЕДЛОЖенИЕ 1. Множсество неэквивалентных общих морфизмов степени $N$ с одной и той же дискриминантной кривой $B$ находится во взаимно однозначном соответствии с множеством эпиморфизмов $\varphi: \pi_{1}\left(\mathbb{P}^{2} \backslash B\right) \rightarrow \mathfrak{S}_{N}$ ( с точностью до внутренних автоморфизмов группь $\left.\mathfrak{S}_{N}\right)$, удовлетворяющих следующим условиям:

(i) для каждой геометрической образующей $\gamma$ ее образ $\varphi(\gamma)$ является транспозичией в $\mathfrak{S}_{N}$;

(ii) для каждого каспа si группа $\varphi\left(G_{i}\right)$ порождена двумя транспозициями и изоморфна группе $\mathfrak{S}_{3}$;

(iii) для каждого ноуда $s_{i}$ группа $\varphi\left(\Gamma_{i}\right)$ порожсдена двумя коммутирующими транспозициями и изоморфна группе $\mathfrak{S}_{2} \times \mathfrak{S}_{2}$. 
ДоКАЗАТЕЛЬСТво. Хорошо известно, что каждый гомоморфизм $\varphi: \pi_{1} \rightarrow \mathfrak{S}_{N}$ определяет конечный морфизм $f: S \rightarrow \mathbb{P}^{2}$ степени $N$, неразветвленный над дополнением к $B$ и такой, что $S$ - нормальная поверхность, и обратно.

Условие (i) эквивалентно условию: $f^{*}(B)=2 R+C$, где $R$ - неприводимая и $C$ - приведенная кривые (см., например, [14] и [16]).

Условия (ii) и (iii) эквивалентны условию: $S$ и $R$ неособы в точках прообраза $f^{-1}\left(s_{i}\right)$ (см., например, [6]).

Так как $S$ - неприводимая поверхность и $\operatorname{deg} f=N$, то $\varphi\left(\pi_{1}\right)$ действует транзитивно на множестве $\bar{N}=\{1, \ldots, N\}$, а так как $\pi_{1}$ порождена геометрическими образующими, то $\varphi\left(\pi_{1}\right)$ порождена некоторым подмножеством транспозиций. Легко проверить, что подгруппа групшы $\mathfrak{S}_{N}$, порожденная некоторым подмножеством транспозиций, действующих на $\bar{N}$ транзитивно, должна совпадать с $\mathfrak{S}_{N}$. Следовательно, $\varphi$ является эпиморфизмом.

ЗАмЕчАниЕ 1 . В силу предложения 1 если $N=2$, то двулистное накрытие $f: S \rightarrow \mathbb{P}^{2}$, разветвленное вдоль неособой кривой $B \subset \mathbb{P}^{2}$, может также рассматриваться как обший морфизм.

ЗАмечАниЕ 2 . Если $B \subset \mathbb{P}^{2}$ - двойственная кривая к гладкой кубике, то по предложению 1 , используя представление группы $\pi_{1}\left(\mathbb{P}^{2} \backslash B\right)$, полученное в [28], легко показать, что $B$ является дискриминантной кривой в точности четырех неэквивалентных общих морфизмов.

Основываясь на этом замечании, можно построить ряд примеров кривых, являюшихся дискриминантными кривыми неэквивалентных обших морфизмов. Для этого рассмотрим общий морфизм $G: \mathbb{P}^{2} \rightarrow \mathbb{P}^{2}$, заданный тремя однородными формами $\left(G_{1}: G_{2}: G_{3}\right)$ степени $k$. Пусть $B_{G} \subset \mathbb{P}^{2}$ - дискриминантная кривая морфизма $G$, и пусть $C^{\prime}$ - двойственная кривая к гладкой кубике, находящаяся в общем положении с кривой $B_{G}$. Кривая $B=G^{-1}\left(C^{\prime}\right)$ является каспидальной кривой степени $\operatorname{deg} B=6 k^{2}$, рода $g=9 k(k-1)+1$ с $c=9 k^{2}$ каспами.

УТВеРЖДЕНИЕ 1. Кривая $B=G^{-1}\left(C^{\prime}\right) \subset \mathbb{P}^{2}$ является дискриминантной кривой четырех неәквивалентных общих морфизмов.

ДокАЗАТЕЛЬСтво. Утверждение следует из того, что $\pi_{1}\left(\mathbb{P}^{2} \backslash B\right)$ изоморфна $\pi_{1}\left(\mathbb{P}^{2} \backslash C^{\prime}\right)$. Действительно, в [17] доказано, что если кривые $C^{\prime}, B_{G}$ и $\mathbb{P}^{1}$ находятся в обшем положении по отношению друг к другу, то

$$
\pi_{1}\left(\mathbb{P}^{2} \backslash\left(B_{G} \cup C^{\prime} \cup \mathbb{P}^{1}\right)\right) \simeq \pi_{1}\left(\mathbb{P}^{2} \backslash\left(B_{G} \cup \mathbb{P}^{1}\right)\right) \times \pi_{1}\left(\mathbb{P}^{2} \backslash\left(C^{\prime} \cup \mathbb{P}^{1}\right)\right),
$$

где $\mathbb{P}^{1}$ - некоторая прямая в $\mathbb{P}^{2}$ (находящаяся в общем положении с $C^{\prime}$ и $B_{G}$ ). Морфизм $G: \mathbb{P}^{2} \backslash G^{-1}\left(B_{G} \cup \mathbb{P}^{1}\right) \rightarrow \mathbb{P}^{2} \backslash\left(B_{G} \cup \mathbb{P}^{1}\right)$ является неразветвленным накрытием и задается некоторой подгруппой конечного индекса $\Gamma$ в $\pi_{1}\left(\mathbb{P}^{2} \backslash\left(B_{G} \cup \mathbb{P}^{1}\right)\right)$. Поэтому

$$
\pi_{1}\left(\mathbb{P}^{2} \backslash\left(B \cup G^{-1}\left(B_{G} \cup \mathbb{P}^{1}\right)\right)\right) \simeq \pi_{1}\left(\mathbb{P}^{2} \backslash\left(C^{\prime} \cup \mathbb{P}^{1}\right)\right) \times \Gamma .
$$

Легко видеть, что ядро гомоморфизма $\pi_{1}\left(\mathbb{P}^{2} \backslash\left(B \cup G^{-1}\left(B_{G} \cup \mathbb{P}^{1}\right)\right)\right) \rightarrow \pi_{1}\left(\mathbb{P}^{2} \backslash B\right)$ порождается элементами из $\Gamma$ и обходом вокруг кривой $G^{-1}\left(\mathbb{P}^{1}\right)$. Поэтому $\pi_{1}\left(\mathbb{P}^{2} \backslash B\right) \simeq \pi_{1}\left(\mathbb{P}^{2} \backslash C^{\prime}\right)$. Следовательно, число неэквивалентных эпиморфизмов из $\pi_{1}\left(\mathbb{P}^{2} \backslash B\right)$ в симметрические группы совпадает с числом эпиморфизмов из $\pi_{1}\left(\mathbb{P}^{2} \backslash C^{\prime}\right)$. 
1.3. Пусть $s_{j} \in B$ - касп и $(x, y)$ - локальные координаты вокруг $s_{j}$ такие, что $B \cap U_{j}$ задана уравнением $y^{2}=x^{3}$. Выберем окрестность $V_{j} \subset S$ точки $p_{j} \in R$, $f\left(p_{j}\right)=s_{j}$, так, что $f=f_{\mid V_{j}}: V_{j} \rightarrow U_{j}$ - трехлистное накрытие, разветвленное вдоль $R \cap V_{j}$. Хорошо известно, что такое накрытие $f: V_{j} \rightarrow U_{j}=U$ единственно с точностью до эквивалентности и, в частности, $f$ эквивалентно стандартному накрытию $\bar{f}: \bar{V} \rightarrow U$, заданному нормализованным уравнением третьей степени:

$$
\begin{gathered}
\bar{V}=\left\{(w, x, y) \mid(x, y) \in U, \quad w^{3}-3 x w+2 y=0\right\}, \\
\bar{f}(w, x, y)=(x, y) .
\end{gathered}
$$

Многообразие $\bar{V}$ неособо и $(x, w)$ являются локальными координатами в $\bar{V}$. Дивизор ветвления $\bar{R}=\left\{(x, w) \in \bar{V} \mid x-w^{2}=0\right\}$ морфизма $\bar{f}$ является гладкой кривой и $\bar{f}^{-1}(B)=2 \bar{R}+\bar{C}$, где $\bar{C}=\left\{(x, w) \in \bar{V} \mid 4 x-w^{2}=0\right\}$. Отметим, что $\bar{R}$ и $\bar{C}$ касаются друг друга в начале координат $o=(0,0)$ и кратность пересечения $\bar{R}$ и $\bar{C}$ в $о$ равна двум.

Как было отмечено вьше, $\pi_{1}(U \backslash B) \simeq \mathrm{Br}_{3}=\langle a, b \mid a b a=b a b\rangle$. Поэтому $\bar{f}: \bar{V} \rightarrow U$ соответствует гомоморфизму $\bar{\varphi}: \pi_{1}(U \backslash B) \rightarrow \mathfrak{S}_{3}$, заданному на образующих $\bar{\varphi}(a)=(1,2)$ и $\bar{\varphi}(b)=(2,3)$.

Положим $\bar{W}=\left\{\left(w_{1}, w_{2}, w_{3}\right) \mid w_{1}+w_{2}+w_{3}=0\right\}$. Уравнения

$$
\begin{aligned}
x & =-\frac{1}{3}\left(w_{1} w_{2}+w_{1} w_{3}+w_{2} w_{3}\right), \\
y & =-\frac{1}{2} w_{1} w_{2} w_{3} \\
w & =w_{1}
\end{aligned}
$$

определяют морфизмы $\tilde{f}: \bar{W} \rightarrow U$ и $g: \bar{W} \rightarrow \bar{V}$ такие, что $\tilde{f}=\bar{f} \circ g, \operatorname{deg} \tilde{f}=6$ и $\operatorname{deg} g=2$. Морфизм $g$ является двулистным накрытием, разветвленным вдоль $\bar{C}$, $g^{*}(\bar{C})=2 \widetilde{C}_{2}$, где $\widetilde{C}_{2}$ задана в координатах $\left(w_{1}, w_{2}\right)$ уравнением $w_{1}+2 w_{2}=0$, a $g^{*}(\bar{R})=\widetilde{R}+\widetilde{C}_{1}$, где $\widetilde{R}$ и $\widetilde{C}_{1}$ заданы уравнениями $w_{1}=w_{2}$ и $2 w_{1}+w_{2}=0$ соответственно.

Заметим, что $\tilde{f}$ соответствует гомоморфизму $\widetilde{\varphi}: \pi_{1}(U \backslash B) \rightarrow \mathfrak{S}_{6}=\mathfrak{S}\left(\mathfrak{S}_{3}\right)$, индуцированному гомоморфизмом $\bar{\varphi}$.

\section{§ 2. Доказательство теоремы 1}

2.1. Предположим, что существуют два неэквивалентных общих морфизма $\left(S_{1}, f_{1}\right)$ и $\left(S_{2}, f_{2}\right)$ с одной и той же дискриминантной кривой $B, \operatorname{deg} f_{1}=N_{1}$ и $\operatorname{deg} f_{2}=N_{2}$. Положим $f_{1}^{*}(B)=2 R_{1}+C_{1}$ и $f_{2}^{*}(B)=2 R_{2}+C_{2}$. Рассмотрим расслоенное произведение

$$
S_{1} \times_{\mathbb{P}^{2}} S_{2}=\left\{(x, y) \in S_{1} \times S_{2} \mid f_{1}(x)=f_{2}(y)\right\} .
$$

Пусть $X=\widetilde{S_{1}} \widetilde{\mathbb{P}^{2}} S_{2}-$ нормализация многообразия $S_{1} \times_{\mathbb{P}^{2}} S_{2}$. Обозначим через $g_{1}: X \rightarrow S_{1}, g_{2}: X \rightarrow S_{2}$ и $f_{1,2}: X \rightarrow \mathbb{P}^{2}$ соответствуюшие естественные морфизмы. Имеем $\operatorname{deg} g_{1}=N_{2}, \operatorname{deg} g_{2}=N_{1}$ и $\operatorname{deg} f_{1,2}=N_{1} N_{2}$. 
ПРЕДЛОЖЕНИЕ 2. Если $\left(S_{1}, f_{1}\right)$ u $\left(S_{2}, f_{2}\right)$ неәквивалентны, то $X$ - неприводимое многообразие.

ДокАЗАТЕЛЬСтво. Морфизм $f_{1,2}$ соответствует гомоморфизму

$$
\varphi_{1,2}=\varphi_{1} \times \varphi_{2}: \pi_{1} \rightarrow \mathfrak{S}_{N_{1}} \times \mathfrak{S}_{N_{2}} \subset \mathfrak{S}_{N_{1} N_{2}},
$$

где $\varphi_{1}: \pi_{1} \rightarrow \mathfrak{S}_{N_{1}}$ (соответственно $\varphi_{2}$ ) - эпиморфизм, соответствуюший морфизму $f_{1}$ (соответственно $f_{2}$ ). Положим $G=\varphi_{1,2}\left(\pi_{1}\right)$. Группа $G$ как подгруппа в $\mathfrak{S}_{N_{1}} \times \mathfrak{S}_{N_{2}}$ действует на $\bar{N}_{1} \times \bar{N}_{2}$. Без ограничения обшности мы можем предполагать, что для некоторой геометрической образуюшей $\gamma$ ее образ $\varphi_{1,2}(\gamma)=$ $((1,2),(1,2))$ есть произведение транспозиций $(1,2) \in \mathfrak{S}_{N_{i}}$. Пусть $p_{i}: G \rightarrow \mathfrak{S}_{N_{i}}-$ ограничение на $G$ проекции $\operatorname{pr}_{i}: \mathfrak{S}_{N_{1}} \times \mathfrak{S}_{N_{2}} \rightarrow \mathfrak{S}_{N_{i}}$.

Лемма 10. Пусть $G$ - подгруппа в $\mathfrak{S}_{N_{1}} \times \mathfrak{S}_{N_{2}}, N_{i}>2$, такая, ито:

1) $p_{i}: G \rightarrow \mathfrak{S}_{N_{i}}$ является эпиморфизмом для $i=1,2$;

2) $((1,2),(1,2)) \in G$.

Пусть $\mathrm{St}_{(1,1)} \subset G-$ стабилизатор әлемента $(1,1) \in \bar{N}_{1} \times \bar{N}_{2}$. Тогда индекс әруппы $\mathrm{St}_{(1,1)}$ в $G$ равен

$$
\left(G: \operatorname{St}_{(1,1)}\right)=N_{1} N_{2},
$$

за исключением случая, когда $N_{1}=N_{2}=N$ и $G=\Delta \subset \mathfrak{S}_{N} \times \mathfrak{S}_{N}($ с точностью до внутренних автоморфизмов одного из множителей), где $\Delta$ диагональная подгруппа.

ДокАЗАТЕЛЬСТво. Включение $\overline{N_{i}-1} \simeq\left\{2, \ldots, N_{i}\right\} \subset\left\{1,2, \ldots, N_{i}\right\}$ определяет вложение $\mathfrak{S}_{N_{i}-1} \subset \mathfrak{S}_{N_{i}}$. Имеем

$$
\operatorname{St}_{(1,1)}=G \cap\left(\mathfrak{S}_{N_{1}-1} \times \mathfrak{S}_{N_{2}-1}\right) .
$$

Положим

$$
H_{1} \times\left\{e_{2}\right\}=G \cap\left(\mathfrak{S}_{N_{1}} \times\left\{e_{2}\right\}\right), \quad\left\{e_{1}\right\} \times H_{2}=G \cap\left(\left\{e_{1}\right\} \times \mathfrak{S}_{N_{2}}\right),
$$

где $e_{i}$ - единица группы $\mathfrak{S}_{N_{i}}$. Заметим, что ker $p_{2}=H_{1} \times\left\{e_{2}\right\}$ и $\operatorname{ker} p_{1}=\left\{e_{1}\right\} \times H_{2}$. Следовательно, $H_{1} \times\left\{e_{2}\right\}$ и $\left\{e_{1}\right\} \times H_{2}$ - нормальные подгрупшы в $G$. Так как $p_{i}: G \rightarrow \mathfrak{S}_{N_{i}}$ - эпиморфизм для $i=1,2$, то $H_{i}-$ нормальная подгруппа в $\mathfrak{S}_{N_{i}}$.

Хорошо известно, что если $H$ - нормальная подгруппа в $\mathfrak{S}_{N}$, то либо $H=\mathfrak{S}_{N}$, либо $H=\mathfrak{A}_{N}$ - знакопеременная группа, либо $H=\{e\}$, и если $N=4$, то существует еше одна возможность: $H$ - группа Клейна

$$
K_{4}=\{e, \quad(1,2)(3,4), \quad(1,3)(2,4), \quad(1,4)(2,3)\}
$$

Рассмотрим все возможные случаи.

Случай I: $H_{1}=\mathfrak{S}_{N_{1}}$. Так как $p_{2}$ - эпиморфизм, то $G=\mathfrak{S}_{N_{1}} \times \mathfrak{S}_{N_{2}}$. Поэтому $|G|=N_{1}$ ! $N_{2}$ ! и $\left|\mathrm{St}_{(1,1)}\right|=\left(N_{1}-1\right)$ ! $\left(N_{2}-1\right)$ !. Следовательно, $\left(G: \operatorname{St}_{(1,1)}\right)=$ $N_{1} N_{2}$. 
Случай II: $H_{1}=\mathfrak{A}_{N_{1}}$. Так как $p_{2}-$ эпиморфизм и $\operatorname{ker} p_{2}=H_{1} \times\{e\}$, то

$$
|G|=\frac{N_{1} ! N_{2} !}{2} .
$$

Аналогично, если мы рассмотрим $p_{1}$, то мы получим, что $\left|\operatorname{ker} p_{1}\right|=N_{2} ! / 2$, поэтому $H_{2}=\mathfrak{A}_{N_{2}}$. Следовательно, $\left(\sigma_{1}, \sigma_{2}\right) \in G$ тогда и только тогда, когда $\sigma_{1}$ и $\sigma_{2}$ имеют один и тот же знак. Поэтому

$$
\left|\operatorname{St}_{(1,1)}\right|=\frac{\left(N_{1}-1\right) !\left(N_{2}-1\right) !}{2}
$$

и $\left(G: \mathrm{St}_{(1,1)}\right)=N_{1} N_{2}$.

Случай III: $H_{1}=\left\{e_{1}\right\}$. Следовательно, $p_{2}$ является изоморфизмом, и имеются две возможности: либо $H_{2}=\left\{e_{2}\right\}$ и $p_{1}$ - также изоморфизм, либо $H_{2} \neq\left\{e_{2}\right\}$. Если $p_{1}$ и $p_{2}-$ изоморфизмы, то $N_{1}=N_{2}=N$ и $G=\Delta \subset \mathfrak{S}_{N} \times \mathfrak{S}_{N}$ с точностью до автоморфизма одного из сомножителей, а так как $((1,2),(1,2)) \in G$, то этот автоморфизм должен быть внутренним. Если $H_{2} \neq\left\{e_{2}\right\}$, то $p_{1} \circ p_{2}^{-1}: \mathfrak{S}_{N_{2}} \rightarrow \mathfrak{S}_{N_{1}}$ является эпиморфизмом (не изоморфизм). Так как $N_{i}>2$, то $\mathfrak{S}_{N_{2}}$ должен совпадать с $\mathfrak{S}_{4}, \mathfrak{S}_{N_{1}}=\mathfrak{S}_{3}$ и $H_{2}=K_{4}$. Завершение исследования этого случая мы оставляем читателю.

Случай IV: $N_{1}=4$ и $H_{1}=K_{4}$. Случай $H_{2}=\mathfrak{S}_{N_{2}}$ невозможен. Действительно, если мы рассмотрим $p_{1}$, то мы получим $|G|=4$ ! $N_{2}$ !. С другой стороны, если рассмотреть $p_{2}$, то $|G|=4 N_{2}$ !. Противоречие.

Случай $H_{2}=\mathfrak{A}_{N_{2}}$ также невозможен. Действительно, если мы рассмотрим $p_{1}$, то $|G|=4 ! N_{2} ! / 2$. С другой стороны, если рассмотреть $p_{2}$, то $|G|=4 N_{2}$ !. Противоречие.

Случай $H_{2}=\left\{e_{2}\right\}$ совпадает (с точностью до индексации) со случаем III.

Случай, когда $N_{2}=4$ и $H_{2}=K_{4}$, будет оставлен читателю.

Чтобы завершить доказательство предложения 2, отметим, что $\operatorname{deg} f_{1,2}=N_{1} N_{2}$ и существует неприводимая компонента $X_{(1,1)}$ многообразия $X$ такая, что

$$
\operatorname{deg} f_{\mid X_{(1,1)}}=\left(G: \operatorname{St}_{(1,1)}\right) .
$$

Поэтому по лемме $9 \operatorname{deg} f_{\mid X_{(1,1)}}=N_{1} N_{2}$ и, следовательно, $X$ неприводимо всегда, за исключением случая, когда $N_{1}=N_{2}=N$ и $G \simeq \Delta \subset \mathfrak{S}_{N} \times \mathfrak{S}_{N}$. Но исключительный случай соответствует случаю, когда $\left(S_{1}, f_{1}\right)$ и $\left(S_{2}, f_{2}\right)$ эквивалентны.

\section{ПРЕДЛОЖЕНИЕ $3 . X$ - неособая поверхность.}

ДокАЗАТЕЛЬСтво. Нам надо проверить гладкость $X$ только в точках $z \in$ $f_{1,2}^{-1}(B)$ таких, что $p_{1}=g_{1}(z) \in R_{1} \subset S_{1}$ и $p_{2}=g_{2}(z) \in R_{2} \subset S_{2}$. Положим $f_{1,2}(z)=s$ и выберем окрестность $V_{1} \subset S_{1}$ точки $p_{1}$ (соответственно окрестность $V_{2} \subset S_{2}$ точки $\left.p_{2}\right)$ и окрестность $U \subset \mathbb{P}^{2}$ точки $s$ так, что $f_{i}\left(V_{i}\right)=U$ и в выбранных окрестностях существуют локальные голоморфные координаты, в которых уравнения, задающие морфизмы $f_{i}$, имеют простейший вид.

Пусть $s \in B$ - неособая точка кривой $B$ или ноуд. Тогда $f_{i}: V_{i} \rightarrow U$ задается уравнениями

$$
u_{i, 1}^{2}=v_{1}, \quad u_{i, 2}=v_{2},
$$


где $v_{1}=0$ - уравнение кривой $B \cap U$ (или одной из ветвей $B$, если $s-$ ноуд). Следовательно, $V_{1} \times_{U} V_{2}$ в $V_{1} \times V_{2}$ задается уравнениями

$$
u_{1,1}^{2}=u_{2,1}^{2}, \quad u_{1,2}=u_{2,2}
$$

или, эквивалентно,

$$
u_{1,1}= \pm u_{2,1}, \quad u_{1,2}=u_{2,2} .
$$

Следовательно, $V_{1} \times_{U} V_{2}$ состоит из двух неприводимых неособых компонент, одна из которых соответствует знаку “+”, а другая - знаку “-”. Следовательно, нормализация $V_{1} \times_{U} V_{2}$ многообразия $V_{1} \times_{U} V_{2}$ есть несвязное объединение двух неособых поверхностей.

Пусть $s=s_{j} \in B$ - касп и $(x, y)$ - локальные координаты вокруг $s$, выбранные в п. 1.3. Пусть $V_{1} \subset S_{1}$ (соответственно для $S_{2}$ ) - окрестность точки $p_{1}=p_{1, j}$ такая, что $f_{1}=f_{1 \mid V_{1}}: V_{1} \rightarrow U$ - трехлистное накрытие, разветвленное вдоль $R_{1} \cap V_{1}$. Положим $Y=V_{1} \times_{U} V_{2}$, и пусть $\widetilde{Y}-$ нормализация поверхности $Y$. Обозначим через $g_{i}: \widetilde{Y} \rightarrow V_{i}$ и $f_{1,2}: \widetilde{Y} \rightarrow U$ соответствуюшие естественные морфизмы. Так как $\left(V_{1}, f_{1}\right)$ и $\left(V_{2}, f_{2}\right)$ эквивалентны, то $f_{1,2}$ соответствует гомоморфизму

$$
\varphi_{1,2}=\varphi_{1} \times \varphi_{2}: \pi_{1}(U \backslash B) \rightarrow \Delta \subset \mathfrak{S}_{3} \times \mathfrak{S}_{3} \subset \mathfrak{S}_{9},
$$

поэтому $\varphi_{1,2}\left(\pi_{1}(U \backslash B)\right)$ действует на $\overline{9} \simeq \overline{3} \times \overline{3}$. Легко проверить, что имеется ровно две орбиты этого действия: одна из них - орбита точки $(1,1)$, а другая - орбита точки $(1,2)$. Следовательно, $\widetilde{Y}$ является несвязным объединением многообразий $\widetilde{Y}_{(1,1)}$ и $\widetilde{Y}_{(1,2)}$. Легко видеть (см., например, [6, лемма 1.6]), что морфизм $\left(\widetilde{Y}_{(1,1)}, f_{1,2}\right)$ эквивалентен морфизму $(\bar{V}, \bar{f})$ (в обозначениях п. 1.3), a $g_{i}: \widetilde{Y}_{(1,1)} \rightarrow V_{i}$ являются изоморфизмами для $i=1,2$; морфизм $\left(\widetilde{Y}_{(1,2)}, f_{1,2}\right)$ эквивалентен морфизму $(\bar{W}, \tilde{f})$, а оба морфизма $\left(\widetilde{Y}_{(1,2)}, g_{i}\right)$ эквивалентны морфизму $(\bar{W}, g)$. Следовательно, $X$ - неособая поверхность.

ЗАМЕЧАнИЕ 3 . Если $\left(S_{1}, f_{1}\right)$ и $\left(S_{2}, f_{2}\right)$ с одной и той же дискриминантной кривой являются эквивалентными морфизмами, то:

1) $X$ - неособая поверхность;

2) $X$ является несвязным объединением двух неприводимых компонент: $X=$ $X_{(1,1)} \sqcup X_{(1,2)}$, таких, что $g_{i \mid X_{(1,1)}}: X_{(1,1)} \rightarrow S_{i}$ является изоморфизмом, $i=1,2$, и $\operatorname{deg} g_{i \mid X_{(1,2)}}=N-1$, где $N=N_{1}=N_{2}$.

2.2. Пусть $\widetilde{R} \subset X$ - кривая $g_{1}^{-1}\left(R_{1}\right) \cap g_{2}^{-1}\left(R_{2}\right)$, положим также $\widetilde{C}=g_{1}^{-1}\left(C_{1}\right) \cap$ $g_{2}^{-1}\left(C_{2}\right), \widetilde{C}_{1}=g_{1}^{-1}\left(R_{1}\right) \cap g_{2}^{-1}\left(C_{2}\right)$ и $\widetilde{C}_{2}=g_{1}^{-1}\left(C_{1}\right) \cap g_{2}^{-1}\left(R_{2}\right)$.

ПРЕДЛОЖЕНИЕ 4. Имеем

$$
\begin{aligned}
\widetilde{R}^{2} & =2(3 d+g-1)-c, \\
\widetilde{C}_{1}^{2} & =\left(N_{2}-2\right)(3 d+g-1)-c, \\
\widetilde{C}_{2}^{2} & =\left(N_{1}-2\right)(3 d+g-1)-c, \\
\left(\widetilde{R}, \widetilde{C}_{i}\right) & =c \quad \text { для } i=1,2 .
\end{aligned}
$$


ДоКАЗАТЕЛЬСТво. В качестве следствия из локального исследования, проведенного в доказательстве предложения 3 , вытекает, что $\operatorname{deg} g_{1 \mid \widetilde{R}}=2$ и $g_{1 \mid \widetilde{R}}$ является этальньм морфизмом.

Легко видеть, что $\widetilde{R}$ и $\widetilde{C}_{i}$ пересекаются только в точках, лежаших над каспами кривой $B$. Рассмотрим одну из таких точек, скажем $s=s_{j}$, и пусть $p_{i}=p_{i, j} \in$ $R_{i} \cap f^{-1}(s)$. Пусть в обозначениях доказательства предложения $3 U$ - окрестность точки $s$. Легко видеть, что одна из ветвей кривой $\widetilde{R} \cap \widetilde{Y}$ принадлежит $\widetilde{Y}_{(1,1)}$, а другая принадлежит $\tilde{Y}_{(1,2)}$. Так как $\left(\tilde{Y}_{(1,1)}, f_{1,2}\right)$ и $\left(V_{i}, f_{i}\right)$ эквивалентны, то $\widetilde{Y}_{(1,1)} \cap \widetilde{C}_{i}=\varnothing$ для $i=1,2$. Рассмотрим $\widetilde{Y}_{(1,2)} \cap \widetilde{R}_{\text {и }} \widetilde{Y}_{(1,2)} \cap \widetilde{C}_{i}$. Так как каждый морфизм $\left(\widetilde{Y}_{(1,2)}, g_{i}\right)$ эквивалентен морфизму $(\bar{W}, g)((\bar{W}, g)$ был определен в п. 1.3), то мы можем отождествить $\left(\widetilde{Y}_{(1,2)}, g_{1}\right)$ с $(\bar{W}, g)$. Тогда $\widetilde{Y}_{(1,2)} \cap \widetilde{R}, \widetilde{Y}_{(1,2)} \cap \widetilde{C}_{1}$ и $\widetilde{Y}_{(1,2)} \cap \widetilde{C}_{2}$ могут быть отождествлены соответственно с $\widetilde{R}, \widetilde{C}_{1}$ и $\widetilde{C}_{2} \subset \bar{W}$. Так как в окрестности $\bar{W}$ кратность пересечения кривых $\widetilde{R}$ и $\widetilde{C}_{i}$ равна 1 , то $\left(\widetilde{R}, \widetilde{C}_{i}\right)=c$.

Чтобы вычислить $\widetilde{R}^{2}$, рассмотрим снова локальный случай. Пусть $g: \bar{W} \rightarrow V$ двулистное накрытие, заданное в локальных аналитических координатах уравнениями

$$
w_{1}^{2}=v_{1}, \quad w_{2}=v_{2}
$$

Обозначим через $C \subset V$ кривую, заданную уравнением $v_{1}=0$, и через $R$ кривую, заданную уравнением $v_{2}^{2}=v_{1}$. Тогда $C$ является кривой ветвления, $g^{*}(C)=2 \widetilde{C}_{2}$ и $g^{*}(R)=\widetilde{R}+\widetilde{C}_{1}$, где $\widetilde{C}_{2}$ задана уравнением $w_{1}=0$, а $\widetilde{R}$ и $\widetilde{C}_{1}$ заданы уравнениями $w_{2}= \pm w_{1}$. Пусть $\sigma: \widetilde{V} \rightarrow V$ - композиция двух $\sigma$-процессов с центрами в точках такая, что $\sigma^{-1}(R+C)=R+C+L_{1}+L_{2}$ - дивизор с нормальными пересечениями, где $L_{1}$ - исключительный дивизор первого $\sigma$-процесса, $L_{2}$ - исключительньй дивизор второго $\sigma$-процесса, и для простоты обозначений мы снова обозначим через $R$ и $C$ соответственно собственные прообразы кривых $R \subset V$ и $C \subset V$. Так как мы осушествили два $\sigma$-процесса с центрами в точках, лежаших на $R$, индекс самопересечения $R^{2}$ уменьшится на 2 (если $R$ рассматривается как полная кривая). Мы можем сделать два $\sigma$-процесса $\widetilde{\sigma}: \widetilde{W} \rightarrow \bar{W}$ (первый с центром в начале координат, а второй с центром в точке пересечения собственного прообраза кривой $\left\{w_{2}=0\right\}$ и исключительного дивизора первого $\sigma$-процесса). Легко проверить, что мы снова получаем морфизм $\bar{g}: \widetilde{W} \rightarrow \widetilde{V}$. Так как мы сделали только один $\sigma$-процесс с центром в точке, лежашей на $\widetilde{W}$, то $\widetilde{W^{2}}$ уменьшится на 1 . Кроме того, $\bar{g}_{\mid \widetilde{R}}: \widetilde{R} \rightarrow R$ является изоморфизмом (локально) и $\bar{g}$ не разветвлен ни в одной из точек, лежащих на $\widetilde{R}$.

Проведенное вьше исследование позволяет нам вычислить $\widetilde{R}^{2}$. Действительно, делая в каждой точке $p_{1, j} \in R_{1}$ два $\sigma$-процесса, как было описано выше, $R_{1}^{2}$ уменьшится на $2 c$. Делая в каждой точке из $g_{1}^{-1}\left(p_{1, j}\right) \cap \widetilde{R}$ два $\sigma$-процесса или так же, как и выше, или если окрестность рассматриваемой точки изоморфна многообразию $\widetilde{Y}_{(1,1)}$, то мы делаем $\sigma$-процессы так, как мы делали это в $V_{1}$, отождествив их в виду того, что $g_{1}: \widetilde{Y}_{(1,1)} \rightarrow V_{1}$ является изоморфизмом. После осушествления всех этих $\sigma$-процессов $\widetilde{R}^{2}$ уменьшится на $3 c$, и мы можем найти окрестность $\widetilde{V}_{1}$ собственного прообраза кривой $R_{1}$ и окрестность $\widetilde{W}$ собственного прообраза кривой $\widetilde{R}$ такие, что ограничение $\widetilde{g}_{1 \mid \widetilde{W}}: \widetilde{W} \rightarrow \widetilde{V}$ полученного морфизма $\widetilde{g}_{1}$ на $\widetilde{W}$ является 
неразветвленным двулистным накрытием. Поэтому

$$
\widetilde{R}^{2}-3 c=2\left(R_{1}^{2}-2 c\right)
$$

Следовательно, применяя лемму 4 , получим $\widetilde{R}^{2}=2(3 d+g-1)-c$.

Так как $\operatorname{deg} g_{1}=N_{2}$, то

$$
N_{2} R_{1}^{2}=\left(\widetilde{R}+\widetilde{C}_{1}, \widetilde{R}+\widetilde{C}_{1}\right)=\widetilde{R}^{2}+2\left(\widetilde{R}, \widetilde{C}_{1}\right)+\widetilde{C}_{1}^{2}
$$

Следовательно,

$$
\widetilde{C}_{1}^{2}=\left(N_{2}-2\right)(3 d+g-1)-c .
$$

Предложение 4 доказано.

2.3. Чтобы завершить доказательство теоремы 1 , применим теорему Ходжа об индексе. Так как по следствию 1 индекс самопересечения $\widetilde{R}^{2}$ больше нуля, то

$$
\left|\begin{array}{cc}
\widetilde{R}^{2} & \left(\widetilde{R}, \widetilde{C}_{i}\right) \\
\left(\widetilde{C}_{i}, \widetilde{R}\right) & \widetilde{C}_{i}^{2}
\end{array}\right|=\left|\begin{array}{cc}
2(3 d+g-1)-c & c \\
c & \left(N_{j}-2\right)(3 d+g-1)-c
\end{array}\right| \leqslant 0,
$$

т.e.

$$
2\left(N_{j}-2\right)(3 d+g-1)^{2}-N_{j}(3 d+g-1) c \leqslant 0 .
$$

Поэтому

$$
N_{j}[2(3 d+g-1)-c] \leqslant 4(3 d+g-1) .
$$

Следовательно, если сушествуют два неэквивалентных общих морфизма $\left(S_{1}, f_{1}\right)$ и $\left(S_{2}, f_{2}\right)$, то их степени должны удовлетворять неравенству

$$
N_{j} \leqslant \frac{4(3 d+g-1)}{2(3 d+g-1)-c}
$$

\section{§ 3. Единственность общего морфизма} для некоторых типов дискриминантных кривых

Запишем неравенство (1) в виде

$$
N[2(3 d+g-1)-c]-4(3 d+g-1)>0
$$

3.1. ДОКАЗАТЕЛЬСТВО ТЕОРЕМЫ 2. Положим $k=K_{S}^{2}, L^{2}=a$ и $\left(K_{S}, L\right)=b$. Отметим, что $a>0$. Если $E=m L$, то

$$
N=\operatorname{deg} f=m^{2} a .
$$

Имеем $K_{S}=f^{*}\left(K_{\mathbb{P}^{2}}\right)+R=-3 E+R$, т.е. $R=K_{S}+3 m L$. Следовательно,

$$
R^{2}=9 m^{2} a+6 m b+k,
$$

4

Серия математическая, №6 $\left(K_{S}, R\right)=3 m b+k$. 
По формуле присоединения имеем

$$
2(g-1)=\left(K_{S}+R, R\right)=9 m^{2}+9 m b+2 k
$$

и по лемме 4

$$
3 d+g-1=9 m^{2} a+6 m b+k .
$$

Из (7), (8) и леммы 7 следует, что

$$
c=12 m^{2} a+9 m b+2 k-e(S) .
$$

Подставим (7), (9) и (10) в (6). Имеем

$$
\begin{aligned}
m^{2} a & {\left[6 m^{2} a+3 m b+e(S)\right]-4\left(9 m^{2} a+6 m b+k\right) } \\
& =m^{2} a\left[6 m^{2} a+3 m b+e(S)-36 a-\frac{24 b}{m a}-\frac{4 k}{m^{2} a}\right]>0 .
\end{aligned}
$$

Теперь очевидно, что существует такая константа $m_{0}$, что последнее неравенство вьполнено для $m \geqslant m_{0}$.

Доказательство теоремы в случае $L=K_{S}$ совпадает с доказательством теоремы 3 .

3.2. ДОКАЗАТЕЛЬСТВО ТЕОРЕМЫ 3. Положим $k=K_{S}^{2}$; тогда $E^{2}=m^{2} k$ и поэтому

$$
N=\operatorname{deg} f=m^{2} k \text {. }
$$

Имеем $K_{S}=f^{*}\left(K_{\mathbb{P}^{2}}\right)+R=-3 E+R$, поэтому $R \equiv(3 m+1) K_{S}$. Следовательно, $\operatorname{deg} B=(E, R)=m(3 m+1) k$, т.е.

$$
d=\frac{m(3 m+1) k}{2}
$$

По формуле присоединения $2(g-1)=\left(K_{S}+R, R\right)$. Следовательно,

$$
g-1=\frac{(3 m+2)(3 m+1) k}{2} .
$$

Из (12) и (13) следует, что

$$
3 d+g-1=(3 m+1)^{2} k .
$$

По лемме 8

$$
c=\left(12 m^{2}+9 m+3\right) k-12 p_{a} .
$$

Подставим (11), (14) и (15) в (6). Имеем

$$
\begin{aligned}
m^{2} k & {\left[\left(6 m^{2}+3 m-1\right) k+12 p_{a}\right]-4(3 m+1)^{2} k } \\
& =m^{2} k\left[\left(6 m^{2}+3 m-1\right) k+12 p_{a}-4\left(3+\frac{1}{m}\right)^{2}\right]>0 .
\end{aligned}
$$


Если $m \geqslant 2$, то

$$
\left[\left(6 m^{2}+3 m-1\right) k+12 p_{a}-4\left(3+\frac{1}{m}\right)^{2}\right] \geqslant 29 k+12 p_{a}-49>0,
$$

а так как $k \geqslant 1$ и $p_{a} \geqslant 1$, то последнее неравенство не выполняется только при $k=1$ и $p_{a}=1$. В исключительном случае если $m \geqslant 3$, то

$$
\left[\left(6 m^{2}+3 m-1\right) k+12 p_{a}-4\left(3+\frac{1}{m}\right)^{2}\right]>62+12-45>0 .
$$

Случай $m=2, k=p_{a}=1$ невозможен. Действительно, в этом случае из (12)-(15) мы получим, что $\operatorname{deg} B=14, g=29, c=57$ и число ноудов $n$ должно быть неотрицательным. Но по формуле присоединения

$$
n=\frac{1}{2}(\operatorname{deg} B-1)(\operatorname{deg} B-2)-g-c=13 \cdot 6-29-57<0 .
$$

Противоречие. Следовательно, неравенство (1) для $m \geqslant 2$ выполнено всегда.

Если $m=1$, то

$$
\left[\left(6 m^{2}+3 m-1\right) k+12 p_{a}-4\left(3+\frac{1}{m}\right)^{2}\right]=8 k+12 p_{a}-64>0,
$$

и, следовательно, неравенство (1) эквивалентно неравенству

$$
2 k+3 p_{a}>16 .
$$

Так как $k=E^{2}=N \geqslant 3$, то последнее неравенство не выполняется только для 1) $k=3, p_{a} \leqslant 3$; 2) $k=4, p_{a} \leqslant 2$; 3$) k=5, p_{a} \leqslant 2$; 4$) k=6, p_{a}=1$.

Покажем, что все исключительные случаи реально не существуют. Действительно, в случае 1$)$ и 2) из (12)-(15) следует, что $\operatorname{deg} B=4 k, g=10 k+1$, $c=12\left(2 k-p_{a}\right)$ и число ноудов $n$ должно быть неотрицательно. Но по формуле присоединения

$$
\begin{aligned}
n & =\frac{1}{2}(\operatorname{deg} B-1)(\operatorname{deg} B-2)-g-c \\
& =(4 k-1)(2 k-1)-10 k-1-24 k+12 p_{a} \\
& =4\left(2 k^{2}-10 k+3 p_{a}\right)<0
\end{aligned}
$$

для $k=3, p_{a} \leqslant 3$ и $k=4, p_{a} \leqslant 2$.

Во всех исключительных случаях, так как $m=1$, должно выполняться неравенство $p_{g} \geqslant 3$, где $p_{g}$ - геометрический род поверхности $S$. Следовательно, $S-$ иррегулярная поверхность, и согласно [8] не сушествует иррегулярной поверхности основного типа $S$ с $K_{S}=5$ и $p_{g} \geqslant 3$. 
Покажем, что морфизм $f: S \rightarrow \mathbb{P}^{2}$ иррегулярной поверхности $S$ с $K_{S}=6$ и $p_{g} \geqslant 3$ не является общим морфизмом ${ }^{1}$. Действительно, согласно [8] в этом случае $S$ изоморфна симметрическому квадрату $C^{(2)}$ кривой $C$ рода $g=3$, а рассматриваемый морфизм $f: S \rightarrow \mathbb{P}^{2}$ совпадает с каноническим морфизмом $\Phi_{\left|K_{C^{(2)}}\right|}$ : $C^{(2)} \rightarrow \mathbb{P}^{2}=X$. Как известно, кривая $C$ изоморфна плоской кривой $C \subset \mathbb{P}^{2}$ степени 4. Легко проверить, что в терминах вложения $C \subset \mathbb{P}^{2}$ пространство $X$ может быть отождествлено с двойственной плоскостью $\mathbb{P}^{2 *}$, а морфизм $\Phi_{\left|K_{C^{(2)}}\right|}$ отождествлен с морфизмом, переводяшим точку $(p, q) \in C^{(2)}$ в точку $l_{p, q} \in \mathbb{P}^{2 *}$, где $l_{p, q}-$ прямая в $\mathbb{P}^{2}$, проходящая через точки $p$ и $q$. Следовательно, кривая ветвления $B$ морфизма $\Phi_{\left|K_{C^{(2)}}\right|}$ совпадает с двойственной кривой $C^{*}$ к $C$. Морфизм $\Phi_{\left|K_{C^{(2)}}\right|}$ может быть разложен в композицию двух морфизмов: морфизма факторизации $\pi: C^{(2)} \rightarrow C^{(2)} / i$ и некоторого морфизма $\psi: C^{(2)} / i \rightarrow \mathbb{P}^{2 *}$, где $C^{(2)} / i-$ факторпространство по действию инволюции $i: C^{(2)} \rightarrow C^{(2)}$, переводящей точку $(p, q) \in C^{(2)}$ в $\left(p^{\prime}, q^{\prime}\right)$, где $\left(p^{\prime}, q^{\prime}\right)$ - дополнительная к $(p, q)$ пара точек пересечения кривой $C$ с прямой $l_{p, q}$. Поэтому $\Phi_{\mid K_{C}(2)} \mid$ не может быть общим морфизмом, так как разложение $\Phi_{\left|K_{C^{(2)}}\right|}=\psi \circ \pi$ не совместимо с условием (iv) в определении общего морфизма.

3.3. ДОКАЗАТЕЛЬСТво тЕОРЕМЫ 4 . Положим $k=K_{S}^{2}$; тогда $E^{2}=m^{2} k$ и поэтому

$$
N=\operatorname{deg} f=m^{2} k
$$

Имеем $K_{S}=f^{*}\left(K_{\mathbb{P}^{2}}\right)+R=-3 E+R$, поэтому $R \in\left|(-3 m+1) K_{S}\right|$. Следовательно, $\operatorname{deg} B=(E, R)=m(3 m-1) k$, т.е.

$$
d=\frac{m(3 m-1) k}{2} .
$$

По формуле присоединения $2(g-1)=\left(K_{S}+R, R\right)$. Следовательно,

$$
g-1=\frac{(3 m-2)(3 m-1) k}{2} .
$$

Из (17) и (18) следует, что

$$
3 d+g-1=(3 m-1)^{2} k .
$$

По лемме 8

$$
c=\left(12 m^{2}-9 m+3\right) k-12 .
$$

Подставим $(16),(19)$ и (20) в (6). Имеем

$$
\begin{aligned}
m^{2} k & {\left[2(3 m-1)^{2} k-\left(12 m^{2}-9 m+3\right) k+12\right]-4(3 m-1)^{2} k } \\
& =m^{2} k\left[\left(6 m^{2}-3 m-1\right) k+12-4\left(3-\frac{1}{m}\right)^{2}\right] \\
& =m^{2} k\left[\left(6\left(m-\frac{1}{4}\right)^{2}-\frac{11}{8}\right) k+12-4\left(3-\frac{1}{m}\right)^{2}\right]>0 .
\end{aligned}
$$

\footnotetext{
${ }^{1}$ Доказательство этого утверждения принадлежит $\Phi$. Катанезе.
} 
Если $m \geqslant 3$, то неравенство (1) выполнено, так как

$$
\left[\left(6 m^{2}-3 m-1\right) k+12-4\left(3-\frac{1}{m}\right)^{2}\right]>26 k+12-36>0 .
$$

Если $m=2$, то неравенство (1) выполнено, так как

$$
\left[\left(6 m^{2}-3 m-1\right) k+12-4\left(3-\frac{1}{m}\right)^{2}\right]=17 k+12-25>0 .
$$

Если $m=1$, то неравенство (1) также выполнено, так как в этом случае $k \geqslant 3$, поэтому

$$
\left[\left(6 m^{2}-3 m-1\right) k+12-4\left(3-\frac{1}{m}\right)^{2}\right]=2 k+12-16>0 .
$$

3.4. ДОКАЗАТЕЛЬСтво теОремЫ 5 . Пусть $f^{-1}\left(\mathbb{P}^{1}\right)=E \in\left|a L_{1}+b L_{2}\right|$, где $L_{1}$ и $L_{2}$ - естественные образуюшие групшы $\mathrm{Pic} S$. Без ограничения обшности мы можем предполагать, что $a \geqslant b>0$. Тогда

$$
N=\operatorname{deg} f=2 a b .
$$

Имеем $K_{S}=-2 L_{1}-2 L_{2}$ и $K_{S}=f^{*}\left(K_{\mathbb{P}^{2}}\right)+R=-3 E+R$, поэтому

$$
R \in\left|(3 a-2) L_{1}+(3 b-2) L_{2}\right| .
$$

Следовательно, $\operatorname{deg} B=(E, R)=a(3 b-2)+b(3 a-2)$, т.е.

$$
d=3 a b-a-b .
$$

По формуле присоединения $2(g-1)=\left(K_{S}+R, R\right)$. Следовательно,

$$
g-1=9(a b-a-b)+8 .
$$

Из (22) и (23) следует, что

$$
3 d+g-1=18 a b-12 a-12 b+8 .
$$

По лемме 7

$$
c=24 a b-18 a-18 b+12 .
$$

Подставим $(21),(24)$ и $(25)$ в (6). Имеем

$$
\begin{aligned}
& 2 a b {[12 a b-6 a-6 b+4]-4(18 a b-12 a-12 b+8) } \\
& \quad=4 a b\left[3 a(b-1)+3 b(a-1)+\frac{12}{b}+\frac{12}{a}-16-\frac{8}{a b}\right]>0 .
\end{aligned}
$$

Если $a \geqslant b \geqslant 2$, то неравенство (1) вьполнено, так как

$$
\begin{aligned}
& 3 a(b-1)+3 b(a-1)+\frac{12}{b}+\frac{12}{a}-16-\frac{8}{a b} \\
& \quad \geqslant 3 a+6(a-1)+\frac{8}{a}+\frac{12}{b}-16=9 a+\frac{8}{a}+\frac{12}{b}-22>0 .
\end{aligned}
$$

Если $a>b=1$, то неравенство (1) также выполнено, так как

$$
\begin{aligned}
& 3 a(b-1)+3 b(a-1)+\frac{12}{b}+\frac{12}{a}-16-\frac{8}{a b} \\
& \quad=3(a-1)+12+\frac{4}{a}-16=3 a+\frac{4}{a}-7>0 .
\end{aligned}
$$

Если $a=b=1$, то $f$ является двулистным накрытием плоскости $\mathbb{P}^{2}$, разветвленным вдоль гладкой коники. 
3.5. ДОКАЗАТЕЛЬСТвО ТЕОРЕм 6-8. Пусть

$$
\operatorname{deg} f=N=E^{2}=2 k
$$

( $E^{2}$ - четное число, так как $2 K_{S}$ тривиален). Имеем $K_{S}=f^{*}\left(K_{\mathbb{P}^{2}}\right)+R=-3 E+R$, поэтому $R \equiv 3 E$. Следовательно, $\operatorname{deg} B=6 k$, т.е.

$$
d=3 k
$$

По формуле присоединения $2(g-1)=R^{2}$. Следовательно,

$$
g-1=9 k .
$$

Из (27) и (28) следует, что

$$
3 d+g-1=18 k \text {. }
$$

По лемме 8

$$
c=24 k-12 p_{a} .
$$

Подставим (26), (29) и (30) в (6). Имеем

$$
2 k\left[12 k+12 p_{a}\right]-72 k=24 k\left[k+p_{a}-3\right]>0,
$$

и $k \geqslant 2$, так как $N>2$. Если $S-$ К3-поверхность, то неравенство (1) выполнено, так как $p_{a}=2$.

Если $S$-поверхность Энриквеса, то $p_{a}=1$, и неравенство (1) также выполнено, за исключением, возможно, случая $k=2$. Для абелевой поверхности $\left(p_{a}=0\right)$ неравенство (1) выполнено, за исключением, возможно, случаев $k=2$ и $k=3$.

Для абелевой поверхности случай $k=2$ невозможен, так как такая кривая $B$ не может существовать. Действительно, в этом случае из (27), (28) и (30) следует, что $\operatorname{deg} B=12, g=19, c=48$. Тогда

$$
n=\frac{1}{2}(\operatorname{deg} B-1)(\operatorname{deg} B-2)-g-c=55-19-48<0,
$$

что невозможно.

Если $S$ - поверхность Энриквеса, то в исключительном случае мы имеем $\operatorname{deg} f=4$. Рассмотрим эпиморфизм $\varphi: \pi_{1}=\pi_{1}\left(\mathbb{P}^{2} \backslash B\right) \rightarrow \mathfrak{S}_{4}$, соответствуюший морфизму $f$. Из (27), (28) и (30) следует, что $\operatorname{deg} B=12, g=19$ и $c=36$, поэтому $n=0$. Следовательно, по предложению 1 эпиморфизм $\varphi^{\prime}: \pi_{1} \rightarrow \mathfrak{S}_{3}$, являюшийся композицией $\varphi$ и естественного эпиморфизма $\mathfrak{S}_{4} \rightarrow \mathfrak{S}_{3}=\mathfrak{S}_{4} / K_{4}$, где $K_{4}$ четверная группа Клейна, соответствует общему морфизму $f^{\prime}: S^{\prime} \rightarrow \mathbb{P}^{2}$ степени 3 с той же самой дискриминантной кривой $B$.

Утверждение о степени общих морфизмов в исключительном случае следует из леммы 5 . 
3.6. ДокАЗАТЕльСтво тЕОРемы 9 . Пусть $S=X\left(m_{1}, \ldots, m_{k}\right) \subset \mathbb{P}^{k+2}-$ полное пересечение мультистепени $\left(m_{1}, \ldots, m_{k}\right), m_{i}>1$. Тогда для общей проекции $f$ на $\mathbb{P}^{2}$

$$
\operatorname{deg} f=N=\prod_{i=1}^{k} m_{i} .
$$

По формуле присоединения имеем $K_{S}=\left(m_{1}+\ldots+m_{k}-k-3\right) E$. Так как $K_{S}=f^{*}\left(K_{\mathbb{P}^{2}}\right)+R=-3 E+R$, то $R=\left(m_{1}+\ldots+m_{k}-k\right) E$. Следовательно, $\operatorname{deg} B=\left(m_{1}+\ldots+m_{k}-k\right) E^{2}$, т.e.

$$
d=\frac{1}{2}\left(\sum_{i=1}^{k}\left(m_{i}-1\right)\right) \prod_{i=1}^{k} m_{i}
$$

По формуле присоединения $2(g-1)=R^{2}+\left(R, K_{S}\right)$. Следовательно,

$$
g-1=\frac{1}{2}\left(\sum_{i=1}^{k}\left(m_{i}-1\right)\right)\left(2 \sum_{i=1}^{k}\left(m_{i}-1\right)-3\right) \prod_{i=1}^{k} m_{i} .
$$

Из (32) и (33) следует, что

$$
3 d+g-1=\left(\sum_{i=1}^{k}\left(m_{i}-1\right)\right)^{2} \prod_{i=1}^{k} m_{i} .
$$

По лемме 7

$$
\begin{aligned}
c & =3 N+2(g-1)-e(S) \\
& =3 \prod_{i=1}^{k} m_{i}+\left(\sum_{i=1}^{k}\left(m_{i}-1\right)\right)\left(2 \sum_{i=1}^{k}\left(m_{i}-1\right)-3\right) \prod_{i=1}^{k} m_{i}-e(S) .
\end{aligned}
$$

Подставим (31), (34) и (35) в (6). Имеем

$$
\prod_{i=1}^{k} m_{i}\left[3 \prod_{i=1}^{k} m_{i}\left(\sum_{i=1}^{k}\left(m_{i}-1\right)\right)+e(S)-4\left(\sum_{i=1}^{k}\left(m_{i}-1\right)\right)^{2}\right]>0 .
$$

Если $k \geqslant 2$, то

$$
\begin{array}{r}
{\left[3 \prod_{i=1}^{k} m_{i}\left(\sum_{i=1}^{k}\left(m_{i}-1\right)\right)+e(S)-4\left(\sum_{i=1}^{k}\left(m_{i}-1\right)\right)^{2}\right]} \\
>\left(\sum_{i=1}^{k}\left(m_{i}-1\right)\right)\left[3 \prod_{i=1}^{k} m_{i}-4\left(\sum_{i=1}^{k}\left(m_{i}-1\right)\right)\right]>0
\end{array}
$$

и, очевидно, последнее неравенство является верным.

Если $k=1$, то $e(S)=m_{1}^{3}-4 m_{1}^{2}+6 m_{1}$, поэтому

$$
\begin{aligned}
3 & \prod_{i=1}^{k} m_{i}\left(\sum_{i=1}^{k}\left(m_{i}-1\right)\right)+e(S)-4\left(\sum_{i=1}^{k}\left(m_{i}-1\right)\right)^{2} \\
& =3 m_{1}\left(m_{1}-1\right)+m_{1}^{3}-4 m_{1}^{2}+6 m_{1}-4\left(m_{1}-1\right)^{2} \\
& =m_{1}^{3}-5 m_{1}^{2}+11 m_{1}-4 \\
& =\left(m_{1}-2\right)^{3}+\left(m_{1}-2\right)^{2}+3\left(m_{1}-2\right)+6 \geqslant 6>0
\end{aligned}
$$

для $m \geqslant 2$, т.е. неравенство (1) также выполнено в случае $k=1$. 
3.7. ДоКАЗАТЕЛЬСТВо ТЕОРЕМЫ 10 . Пусть $B^{*} \subset \mathbb{P}^{2 *}$ - нодальная кривая рода $g, \operatorname{deg} B^{*}=\delta$ и $B-$ двойственная кривая кривой $B^{*}$. Тогда [6] $B$ - дискриминантная кривая некоторого общего морфизма $f$ степени $\delta$. Действительно, пусть $S$ - нормализация поверхности

$$
X=\left\{\left(\left(x_{1}, x_{2}, x_{3}\right),\left(y_{1}, y_{2}, y_{3}\right)\right) \in \mathbb{P}^{2} \times \mathbb{P}^{2 *} \mid \sum_{i} x_{i} y_{i}=0, \quad\left(y_{1}, y_{2}, y_{3}\right) \in B^{*}\right\}
$$

и $f$ индуцирован проекцией $\mathrm{pr}_{1}: \mathbb{P}^{2} \times \mathbb{P}^{2 *} \rightarrow \mathbb{P}^{2}$. Ясно, что $\left(x_{1}, x_{2}, x_{3}\right) \notin B$, если прямая $\sum_{i} x_{i} y_{i}=0$ не касается $B^{*}$, в этом случае $f^{-1}\left(\left(x_{1}, x_{2}, x_{3}\right)\right)$ имеет точно $\delta$ точек и

$$
N=\operatorname{deg} f=\delta,
$$

поэтому $B$ - кривая ветвления морфизма $f$, и ясно, что $f$ - общий морфизм.

Из формул Плюккера следует, что

$$
\begin{aligned}
& d=\delta+(g-1), \\
& c=3 \delta+6(g-1) .
\end{aligned}
$$

Подставим (36)-(38) в неравенство (1). Имеем

$$
\delta>\frac{4(3 \delta+4(g-1))}{3 \delta+2(g-1)}=8-\frac{12 \delta}{3 \delta+2(g-1)} .
$$

Следовательно, неравенство (1) выполнено для $\delta \geqslant 8$.

Рассмотрим случай $\delta \leqslant 7$. При $\delta=7$ неравенство (1) не вьполняется, если

$$
\frac{12 \delta}{3 \delta+2(g-1)} \leqslant 1 \Longrightarrow 9 \delta \leqslant 2(g-1) \Longrightarrow g \geqslant 33 .
$$

С другой стороны,

$$
g \leqslant \frac{(\delta-1)(\delta-2)}{2}=\frac{6 \cdot 5}{2}<33 .
$$

Следовательно, в случае $\delta=7$ неравенство (1) является верным.

При $\delta=6$ неравенство (1) не выполняется, если

$$
\frac{12 \delta}{3 \delta+2(g-1)} \leqslant 2 \Longrightarrow 3 \delta \leqslant 2(g-1) \Longrightarrow g \geqslant 10 .
$$

С другой стороны,

$$
g \leqslant \frac{(\delta-1)(\delta-2)}{2}=\frac{5 \cdot 4}{2}=10 .
$$

Следовательно, в случае $\delta=6$ неравенство (1) не является верным, только если $\nu=0$, т.е., возможно, существуют два неэквивалентных общих морфизма только в случае, когда $B$ имеет следуюшие инварианты: $\operatorname{deg} B=30, g=10, c=72$, 
$n=324$. Для такой $B$, если существует другой (неэквивалентный морфизму $f$ ) обший морфизм $f_{1}$ степени $\operatorname{deg} f_{1}=N_{1}$, имеем

$$
N_{1} \leqslant \frac{4(3 d+(g-1))}{6 d+2(g-1)-c}=\frac{4(3 \cdot 15+(10-1))}{6 \cdot 15+2(10-1)-72}=6 .
$$

При $\delta=5$ неравенство (1) не вьполняется, если

$$
\frac{12 \delta}{3 \delta+2(g-1)} \leqslant 3 \Longrightarrow \delta \leqslant 2(g-1) \Longrightarrow g \geqslant 4 \text {. }
$$

С другой стороны,

$$
g \leqslant \frac{(\delta-1)(\delta-2)}{2}=\frac{4 \cdot 3}{2}=6 .
$$

Следовательно, в случае $\delta=5$ неравенство (1) не выполняется, если $\nu=0$, или $\nu=1$, или $\nu=2$, т.е., возможно, существуют два неэквивалентных общих морфизма только в следуюших трех случаях:

0) $\operatorname{deg} B=20, g=6, c=45$;

1) $\operatorname{deg} B=18, g=5, c=39$;

2) $\operatorname{deg} B=16, g=4, c=33$.

Во всех случаях 0) - 2), если существуют два неэквивалентных общих морфизма $f$ и $f_{1}$ с одной и той же дискриминантной кривой $B$, вычисления, аналогичные описанным вьше, приводят к $\operatorname{deg} f_{1}=N_{1} \leqslant 5$.

При $\delta=4$ имеем $g \leqslant 3$. Если $g=3$, то $\operatorname{deg} B=12$ и $c=24$. Легко проверить, что если существуют два неэквивалентных общих морфизма $f$ и $f_{1}$ с такой дискриминантной кривой $B$, то $\operatorname{deg} f_{1}=N_{1} \leqslant 5$. Покажем, что такая кривая не может быть дискриминантной кривой общего морфизма $f_{1}: S_{1} \rightarrow \mathbb{P}^{2}$ степени 5 . Действительно, в этом случае из лемм $6-8$ следует, что $K_{S_{1}}^{2}=-7, e\left(S_{1}\right)=-5$ и $p_{a}=-1$. Следовательно, $S_{1}-$ линейчатая поверхность над некоторой кривой $C$ рода $g(C)=2$. Пусть $\bar{S}_{1}$ - относительно минимальная модель поверхности $S_{1}$. Тогда $e\left(\bar{S}_{1}\right)=-4$, поэтому $е\left(S_{1}\right) \geqslant-4$. Противоречие.

Случай $g \leqslant 2$ будет рассмотрен в доказательстве теоремы 11 .

3.8. ДОКАЗАТЕЛЬСТВО ТЕОРЕМЫ 11 . В следующем пункте мы докажем теорему 12. По этой теореме гипотеза Кизини выполнена для кривой $B$ рода $g \leqslant 1$, и если в случае $g=2$ существуют два неэквивалентных общих морфизма $f_{1}$ и $f_{2}$, то $d \leqslant 3$.

Рассмотрим случай $g=2$ и $d=3$. В этом случае неравенство, противоположное неравенству (1), принимает следуюший вид:

$$
N_{i} \leqslant \frac{40}{20-c}
$$

Следовательно, если $N_{i} \geqslant 5$, то

$$
5 \leqslant \frac{40}{20-c} \Longleftrightarrow 12 \leqslant c \leqslant 19 .
$$


С другой стороны,

$$
c=(2 d-1)(d-1)-g-n=8-n \leqslant 8 .
$$

Противоречие.

Кривая $B$ с инвариантами $g=2$ и $\operatorname{deg} B=4$ (т.е. $d=2$ ) не может быть дискриминантной кривой. Действительно, в этом случае либо $c=1$, либо $n=1$, что противоречит следствию 2.

Рассмотрим случай $g=3$. По теореме 12 если сушествуют два неэквивалентных обших морфизма $f_{1}$ и $f_{2}$ таких, что $N_{1} \geqslant 5$, то $d \leqslant 6$. Из лемм 2 и 3 следует, что

$$
\frac{3}{2} d+1 \leqslant c \leqslant 3 d+6,
$$

и неравенство, противоположное неравенству (1), принимает следуюший вид:

$$
5 \leqslant \frac{12 d+8}{6 d+4-c} \Longleftrightarrow \frac{18 d+12}{5} \leqslant c \leqslant 6 d+3 .
$$

Если $d=6$, то из $(39)$ следует, что $10 \leqslant c \leqslant 24$. С другой стороны, из (40) имеем $c \geqslant 24$ и, следовательно, $c=24$ и $n=28$. Этот случай уже был рассмотрен в доказательстве теоремы 10.

Если $d=5$, то из $(39)$ следует, что $9 \leqslant c \leqslant 21$. С другой стороны, из (40) имеем $c \geqslant 21$ и, следовательно, $c=21$ и $n=12$. Из формул Плюккера следует, что $\delta=10 \cdot 9-2 \cdot 12-3 \cdot 21=3$. Получаем противоречие с $g=3$.

Если $d=4$, то из $(39)$ следует, что $7 \leqslant c \leqslant 18$. С другой стороны, из (40) имеем $c \geqslant 17$ и из следствия 2 получаем, что $c=18$ и $n=0$. Из формул Плюккера следует, что $\delta=8 \cdot 7-3 \cdot 21 \leqslant 0$. Противоречие.

Если $d=3$, то из (39) следует, что $6 \leqslant c \leqslant 15$. С другой стороны, из (40) имеем $c \geqslant 14$ и из следствия 2 получаем, что $c=15$, что противоречит неравенству $g=(2 d-1)(d-1)-c-n \geqslant 0$.

Если $d=2$, т.е. $\operatorname{deg} B=4$, то $B$ - неособая кривая. Следовательно, $B$ не может быть дискриминантной кривой.

3.9. ДокАЗАТЕЛЬСтво теоремы 12 . Рассмотрим снова неравенство, противоположное неравенству (1):

$$
N_{i} \leqslant \frac{4(3 d+(g-1))}{6 d+2(g-1)-c} .
$$

Следовательно, если существуют два неэквивалентных общих морфизма таких, что один из них имеет степень $N_{i} \geqslant 5$, то

$$
\begin{aligned}
& \frac{4(3 d+(g-1))}{6 d+2(g-1)-c} \geqslant 5 \\
& \quad \Longrightarrow 5 c \geqslant 18 d+6(g-1) \Longrightarrow \quad(\text { лемма } 2) \\
& \quad \Longrightarrow 15 d+15(g-1) \geqslant 18 d+6(g-1) \\
& \quad \Longleftrightarrow 3(g-1) \geqslant d .
\end{aligned}
$$




\section{§ 4. Канонические дискриминантные кривые}

4.1. Назовем кривую $B$ (m-) канонической дискриминантной кривой, если $B$ - дискриминантная кривая общего морфизма $f: S \rightarrow \mathbb{P}^{2}$, заданного линейной системой $\{E\} \subseteq\left|m K_{S}\right|, m \in \mathbb{N}$.

Пусть $B \subset \mathbb{P}^{2}$ - каспидальная кривая четной степени $2 d$, и пусть $\tilde{\nu}: R \rightarrow B$ - ее нормализация. Положим $\mathfrak{e}=\tilde{\nu}^{-1}\left(\mathbb{P}^{1} \cap B\right), \mathfrak{c}=2 \sum^{\prime} \tilde{\nu}^{-1}\left(s_{i}\right)$ и $\mathfrak{n}=\sum^{\prime \prime} \tilde{\nu}^{-1}\left(s_{i}\right)$, где $\sum^{\prime}$ (соответственно $\left.\sum^{\prime \prime}\right)$ - суммирование по всем каспам $s_{i} \in B$ (соответственно по всем ноудам).

ПРЕДЛОЖЕНИЕ 5. Пусть В и $R$ - такие кривые, как описано выше. Если $B$ - каноническая дискриминантная кривая, то:

(i) $\frac{2 d}{g-3 d-1}:=m \in \mathbb{N}$;

(ii) $\frac{(g-3 d-1)^{2}}{3 d+g-1}:=k \in \mathbb{N}$

(iii) $\frac{4 d^{2}}{3 d+g-1}:=N \in \mathbb{N}$;

(iv) $N+\frac{3 g-3-9 d-c}{12}:=p_{a} \in \mathbb{N}$;

(v) существует дивизор $\mathfrak{k} \in \operatorname{Pic} R$ такой, что $K_{R}=(3 m+2) \mathfrak{k} u \mathfrak{e}=m \mathfrak{k}$.

Кроме того,

$$
\operatorname{dim} H^{0}\left(R, \mathscr{O}_{R}(r \mathfrak{k})\right)=\frac{r(r-1)}{2} k+p_{a}
$$

$\partial л я r=2, \ldots, 3 m$

(vi) $\mathfrak{c}+\mathfrak{n}=[(2 d-6) m-2] \mathfrak{k}$.

ДоКАЗАТЕЛЬСТво. Из (12) и (13) следует, что

$$
\begin{aligned}
& \left\{\begin{array}{rl}
2 g-2 & =\left(9 m^{2}+9 m+2\right) k \\
6 d & =\left(9 m^{2}+3 m\right) k
\end{array} \Longrightarrow g-1-3 d\right. \\
& =(3 m+1) k \Longrightarrow m=\frac{2 d}{g-3 d-1} \text {. }
\end{aligned}
$$

Поэтому (ii) следует из (12), (iii) следует из (11), а (iv) - из (15).

Элемент $\mathfrak{k}$ Ріс $R$ является ограничением канонического класса $K_{S}$ на $R$. Так как $K_{S}$ обилен, то

$$
\operatorname{dim} H^{1}\left(S, \mathscr{O}_{S}\left(-r K_{S}\right)\right)=0
$$

для $r>0$. Поэтому из точной последовательности

$$
0 \longrightarrow \mathscr{O}_{S}\left((r-3 m-1) K_{S}\right) \longrightarrow \mathscr{O}_{S}\left(r K_{S}\right) \longrightarrow \mathscr{O}_{R}\left(r K_{S}\right) \longrightarrow 0
$$

следует, что $H^{0}\left(S, \mathscr{O}_{S}\left(r K_{S}\right)\right)$ изоморфно пространству $H^{0}\left(R, \mathscr{O}_{R}\left(r K_{S}\right)\right)$ для $1 \leqslant r \leqslant 3 m$. По теореме Римана-Роха

$$
\operatorname{dim} H^{0}\left(S, \mathscr{O}_{S}\left(r K_{S}\right)\right)=\frac{r(r-1)}{2} K_{S}^{2}+p_{a}
$$

для $r>1$. 
Чтобы доказать (vi), раздуем все особые точки кривой $B$. Обозначим композицию этих $c+n \sigma$-процессов через $\sigma: \widetilde{\mathbb{P}^{2}} \rightarrow \mathbb{P}^{2}$, и пусть $L_{i}=\sigma^{-1}\left(s_{i}\right)$ - исключительные кривые, лежащие над $s_{i} \in \operatorname{Sing} B$. Тогда $\sigma^{*}(B)=R+2 \sum L_{i}$, где собственный прообраз $R$ кривой $B$ является неособой кривой, так как все особые точки кривой $B$ суть каспы и ноуды. Имеем

$$
\begin{aligned}
K_{\widetilde{\mathbb{P}^{2}}} & =-3 \sigma^{*}\left(\mathbb{P}^{1}\right)+\sum L_{i}, \\
R & =\operatorname{deg} B \sigma^{*}\left(\mathbb{P}^{1}\right)-2 \sum L_{i} .
\end{aligned}
$$

Следовательно, по формуле присоединения

$$
\mathscr{O}_{R}\left(K_{R}\right)=\mathscr{O}_{R}\left(K_{\widetilde{\mathbb{P}^{2}}}+R\right)=\mathscr{O}_{R}\left((\operatorname{deg} B-3) \sigma^{*}\left(\mathbb{P}^{1}\right)-\sum L_{i}\right) .
$$

Но $\mathscr{O}_{R}(\mathfrak{c}+\mathfrak{n})=\mathscr{O}_{R}\left(\sum L_{i}\right)$. Следовательно,

$$
\mathscr{O}_{R}(\mathfrak{c}+\mathfrak{n})=\mathscr{O}_{R}(((2 d-6) m-2) \mathfrak{k}),
$$

так как $\mathscr{O}_{R}\left(\sigma^{*}\left(\mathbb{P}^{1}\right)\right)=\mathscr{O}_{R}(\mathfrak{e})=\mathscr{O}_{R}(m \mathfrak{k})$ и $\mathscr{O}_{R}\left(K_{R}\right)=\mathscr{O}_{R}((3 m+2) \mathfrak{k})$.

ПРЕДЛОЖЕНИЕ 6. Пусть В удовлетворяет условиям (i)-(vi) предложения 5. Если $B$ - дискриминантная кривая общего морфизма $f: S \rightarrow \mathbb{P}^{2}$ степени $\operatorname{deg} f=N$, то $S$ является поверхностью общего типа с обильным каноническим классом, а $f$ задается трехмерной линейной подсистемой в $\left|m K_{S}+\alpha\right|$, где $\alpha \in \operatorname{Pic} S$ - элемент второго порядка. Более того, если $m-$ четное число, то $\alpha=0$.

ДОКАЗАТЕЛЬСТВо. Пусть $f$ задан линейной системой $|E|$. Имеем

$$
E^{2}=N=\frac{4 d^{2}}{3 d+g-1},
$$

$R^{2}=3 d+g-1$ и $(E, R)=2 d$. Тогда

$$
\left|\begin{array}{cc}
E^{2} & (E, R) \\
(R, E) & R^{2}
\end{array}\right|=\left|\begin{array}{cc}
\frac{4 d^{2}}{3 d+g-1} & 2 d \\
2 d & 3 d+g-1
\end{array}\right|=0 .
$$

Следовательно, по теореме Ходжа об индексе

$$
R \equiv \frac{3 d+g-1}{2 d} E \equiv \frac{3 m+1}{m} E .
$$

Tак как $K_{S}=-3 E+R$, то

$$
E \equiv m K_{S}, \quad R \equiv(3 m+1) K_{S},
$$

и если $E=m K_{S}+\alpha$, где $\alpha \equiv 0$, и $\mathscr{O}_{R}\left(K_{S}\right)=\mathscr{O}_{R}(\mathfrak{k}+\beta)$, где $\operatorname{deg} \beta=0$, то

$$
\begin{aligned}
& \mathscr{O}_{R}(E)=\mathscr{O}_{R}(m \beta+\alpha+m \mathfrak{k})=\mathscr{O}_{R}(m \mathfrak{k}), \\
& \mathscr{O}_{R}(R)=\mathscr{O}_{R}\left(K_{S}+3 E\right)=\mathscr{O}_{R}((3 m+1) \beta+3 \alpha+(3 m+1) \mathfrak{k}) .
\end{aligned}
$$

Следовательно, $\mathscr{O}_{R}\left(K_{R}\right)=\mathscr{O}_{R}((3 m+2) \beta+3 \alpha+(3 m+2) \mathfrak{k})$. Поэтому

$$
\mathscr{O}_{R}(m \beta)=\mathscr{O}_{R}(-\alpha), \quad \mathscr{O}_{R}((3 m+2) \beta)=\mathscr{O}_{R}(-3 \alpha) .
$$

Следовательно, $2 \beta=0$ и $\mathscr{O}_{R}(2 \alpha)=\mathscr{O}_{R}$, и если $m$ - четное число, то $\mathscr{O}_{R}(\alpha)=\mathscr{O}_{R}$.

Предложение 6 будет доказано, если мы докажем следующую лемму. 
ЛЕмма 11 (см. дополнение к гл. V в [29]). Пусть $S$ - гладкая проективная поверхность и $i: R \hookrightarrow S-$ гладкая неприводимая кривая. Eсли $\mathscr{O}_{S}(R)$ обилен, то $i^{*}: \mathrm{Pic}^{0} S \rightarrow \operatorname{Pic~} R$ является вложением, где $\mathrm{Pic}^{0} S \subset \mathrm{Pic} S$ - подгруппа численно әквивалентных нулю классов дивизоров.

ДокАЗАТЕЛЬСТво. В коммутативной диаграмме

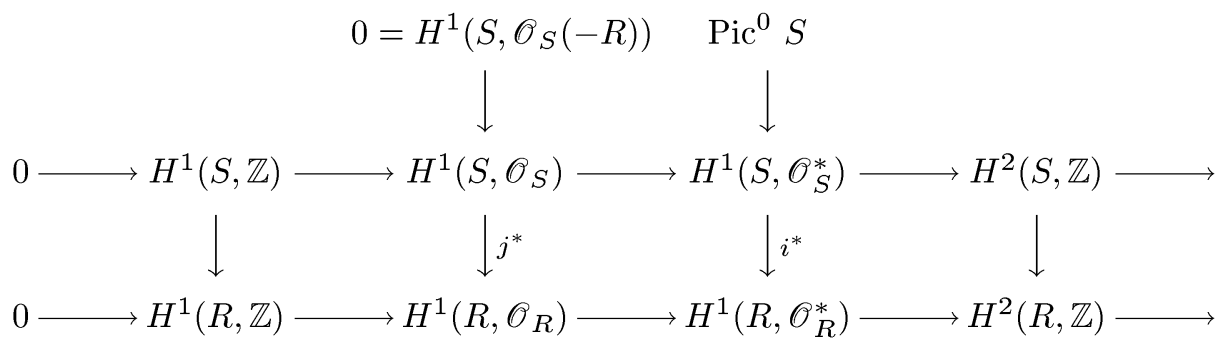

с точными строками морфизм $j^{*}$ является вложением. Следовательно, если для $\alpha \in \operatorname{Pic}^{0} S$ образ $i^{*}(\alpha)=0$, то $r \alpha=0$ для некоторого $r \in \mathbb{N}$, так как Tors $H^{2}(S, \mathbb{Z})$ является конечной абелевой группой и $H^{1}(S, \mathbb{Z}) \rightarrow H^{1}(R, \mathbb{Z})$ является вложением. Элемент $\alpha \in \operatorname{Pic} S$ имеет конечный порядок $r$ и определяет неразветвленное абелево накрытие $\varphi: S_{r} \rightarrow S$ степени $\operatorname{deg} \varphi=r$. Если $\alpha \neq 0$ и $i^{*}(\alpha)=0$, то $\varphi^{-1}(R)$ является несвязным объединением $r$ неприводимых кривых, что противоречит обильности дивизора $R$.

4.2. В обозначениях п. 4.1 рассмотрим естественный гомоморфизм

$$
\mu: \operatorname{Sym}^{2} H^{0}\left(R, \mathscr{O}_{R}(\mathfrak{e})\right) \longrightarrow H^{0}\left(R, \mathscr{O}_{R}(2 \mathfrak{e})\right) .
$$

Ядро ker $\mu$ порождает однородный идеал $I$ в координатном кольце

$$
\mathfrak{R}=\oplus \operatorname{Sym}^{r} H^{0}\left(R, \mathscr{O}_{R}(\mathfrak{e})\right)
$$

однородных функций на проективном пространстве $\mathbb{P}=\mathbb{P} H^{0}\left(R, \mathscr{O}_{R}(\mathfrak{e})\right)$. Положим $S_{I}=\operatorname{Proj} \mathfrak{R} / I$. Нормализация $\tilde{\nu}: R \rightarrow B$ определяет трехмерное подпространство

$$
L=\tilde{\nu}^{*}\left(H^{0}\left(B, \mathscr{O}_{B}\left(\mathbb{P}^{1} \cap B\right)\right)\right) \subseteq H^{0}\left(R, \mathscr{O}_{R}(\mathfrak{e})\right) .
$$

Подпространство $L$ задает проекцию $\mathrm{pr}: \mathbb{P} \rightarrow \mathbb{P}^{2}$ с базисныт множеством $\mathbb{P} L \subset \mathbb{P}$. Пусть $f_{I}: S_{I} \rightarrow \mathbb{P}^{2}$ - ограничение pr на $S_{I}$.

ПРЕДЛОЖЕНИЕ 7. Пусть В удовлетворяет условиям (i)-(vi) предложения 5. Предположим, что $m \geqslant 21$ четно. Кривая $B$ является $m$-канонической дискриминантной кривой тогда и только тогда, когда $S_{I}$ - неособая поверхность степени $\operatorname{deg} S_{I}=N$ и $f_{I}$ является общим морфизмом с дискриминантной кривой $B$. 
ДокАЗАТЕЛЬСТво. Пусть $A$ - очень обильный дивизор на $S$ и $D$ - численно эффективный дивизор. Из [10] следует, что если вложение $S$ в $\mathbb{P}$ задано линейной системой $\left|K_{S}+4 A+D\right|$, то однородный идеал $I(S)$ порождается квадриками.

Согласно [3] $A=5 K_{S}$ является очень обильным дивизором (мы предполагаем, что $K_{S}$ обилен). Следовательно, если $S$ вложено с помощью $\left|m K_{S}\right|, m \geqslant 21$, то $I(S)$ порождается квадриками. С другой стороны, $R \in\left|(3 m+1) K_{S}\right|$ и $R \subset$ $S \subset \mathbb{P}$ вложена с помощью $|m \mathfrak{k}|$. Как уже было упомянуто в доказательстве предложения 5 , морфизм ограничения

$$
H^{0}\left(S, \mathscr{O}_{S}\left(r m K_{S}\right)\right) \longrightarrow H^{0}\left(R, \mathscr{O}_{R}\left(r m K_{S}\right)\right)=H^{0}\left(R, \mathscr{O}_{R}(r \mathfrak{e})\right)
$$

является изоморфизмом для $r=1,2$. Следовательно, множество квадрик, содержащих $S$, совпадает с множеством квадрик, содержащих $R$, и предложение 7 следует из предложений 5 и 6 .

ЗАмечАнИЕ 4. Предложение 7 выполнено также и для нечетных $m \geqslant 21$, если ввиду предложения 6 мы слегка изменим определение $m$-канонической дискриминантной кривой. Более того, предложение 7 есть частный случай более общего утверждения.

ПРЕДЛОЖЕНИЕ $7^{\prime}$. Пусть В удовлетворяет условиям (i)-(iv) предложения 5. Предположим, что $m \geqslant 21$. Кривая В есть дискриминантная кривая морфизма, заданного трехмерной системой $|L|$, где $L$ иисленно әквивалентен т-у каноническому классу тогда и только тогда, когда $S_{I}$ - неособая поверхность степени $\operatorname{deg} S_{I}=N$ и $f_{I}$ - общий морфизм с дискриминантной кривой $B$.

\section{§5. О парах Зариского}

5.1. Множество плоских кривых степени $2 d$ естественным образом параметризовано точками проективного пространства $\mathbb{P}^{d(2 d+3)}$. Подмножество плоских неприводимых каспидальных кривых степени $2 d$ и рода $g$ с $c$ каспами и с некоторым числом (однозначно определяемым этими данными) ноудов соответствует квазипроективному подмногообразию $\mathscr{M}(2 d, g, c) \subset \mathbb{P}^{d(2 d+3)}[27]$. Можно показать, что если две неособые точки одной и той же неприводимой компоненты многообразия $\mathscr{M}_{\text {red }}(2 d, g, c)$ соответствуют кривым $B_{1}$ и $B_{2}$, то пары $\left(\mathbb{P}^{2}, B_{1}\right)$ и $\left(\mathbb{P}^{2}, B_{2}\right)$ диффеоморфны. В частности, в этом случае фундаментальные групшы $\pi_{1}\left(\mathbb{P}^{2} \backslash B_{1}\right)$ и $\pi_{1}\left(\mathbb{P}^{2} \backslash B_{2}\right)$ изоморфны.

Следуюшее предложение является простым следствием предложения 1 и локальных исследований в пп. 1.2 и 1.3.

ПРЕДЛОЖЕНИЕ 8. Пусть $\left(\mathbb{P}^{2}, B_{1}\right)$ u $\left(\mathbb{P}^{2}, B_{2}\right)$ - две гомеоморфные пары $u$ $\psi:\left(\mathbb{P}^{2}, B_{1}\right) \rightarrow\left(\mathbb{P}^{2}, B_{2}\right)$ - гомеоморфизм междуу ними. Если $B_{1}-$ дискриминантная кривая некоторого общего морфизма $\left(S_{1}, f_{1}\right)$, то $B_{2}$ также является дискриминантной кривой некоторого общего морфизма $\left(S_{2}, f_{2}\right)$. Более того, если общий морфизм $\left(S_{1}, f_{1}\right)$ единствен для $B_{1}$, то морфизм $\left(S_{2}, f_{2}\right)$ является единственным общим морфизмом с дискриминантной кривой $B_{2}$, 
кроме того, $S_{1} u S_{2}$ гомеоморфны и, более того, существует коммутативная диаграмма

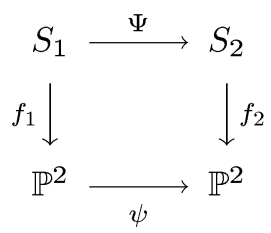

в которой:

(i) $\Psi$ является гомеоморфизмом;

(ii) $\Psi\left(R_{1}\right)=R_{2}$, где $R_{i}-$ кривая ветвления морфизма $f_{i}$.

ДоказАТельство. Гомеоморфизмпар $\psi:\left(\mathbb{P}^{2}, B_{1}\right) \rightarrow\left(\mathbb{P}^{2}, B_{2}\right)$ индуцирует изоморфизм $\psi^{*}: \pi_{1}\left(\mathbb{P}^{2} \backslash B_{2}\right) \rightarrow \pi_{1}\left(\mathbb{P}^{2} \backslash B_{1}\right)$ фундаментальных групп. Согласно предложению 1 множество неэквивалентных общих морфизмов степени $N$ с дискриминантной кривой $B \subset \mathbb{P}^{2}$ взаимно однозначно множеству эпиморфизмов из $\pi_{1}\left(\mathbb{P}^{2} \backslash B\right)$ в симметрическую группу $\Sigma_{N}$, удовлетворяющих некоторым условиям (подробности см. в п. 1.2). Так как для $B_{1} \subset \mathbb{P}^{2}$ вьполнена гипотеза Кизини, то для $\pi_{1}\left(\mathbb{P}^{2} \backslash\right.$ $\left.B_{1}\right)$ существует единственный такой эпиморфизм, и он совпадает с индуцированным $f_{1}$ эпиморфизмом $f_{1 *}: \pi_{1}\left(\mathbb{P}^{2} \backslash B_{1}\right) \rightarrow \Sigma_{N}$, где $N=\operatorname{deg} f_{1}$. Следовательно, для $B_{2}$ также существует, и при том единственный, такой эпиморфизм, и он должен совпадать с $f_{1 *} \circ \psi^{*}=f_{2 *}: \pi_{1}\left(\mathbb{P}^{2} \backslash B_{2}\right) \rightarrow \Sigma_{N}$. Отсюда следует, что гомеоморфизм $\psi:\left(\mathbb{P}^{2}, B_{1}\right) \rightarrow\left(\mathbb{P}^{2}, B_{2}\right)$ можно включить в коммутативную диаграмму

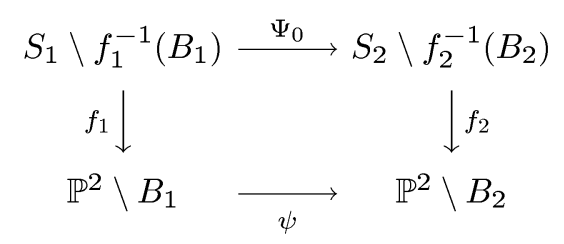

В [16] изложен метод восстановления поверхности $S$ и конечного морфизма $f: S \rightarrow \mathbb{P}^{2}$, разветвленного вдоль $B \subset \mathbb{P}^{2}$, по гомоморфизму $f_{*}: \pi_{1}\left(\mathbb{P}^{2} \backslash B\right) \rightarrow \Sigma_{N}$, основанный на представлении $S$ в виде $N$ экземпляров $\mathbb{P}^{2}$ со “стандартными разрезами", склеенных вдоль этих разрезов (при этом мы должны воспользоваться содержащимся в [15] геометрическим описанием конечного копредставления группы $\pi_{1}\left(\mathbb{P}^{2} \backslash B\right)$ в терминах “теней” и “экранов” ). Применяя этот метод, легко видеть, что гомеоморфизм $\Psi_{0}$ однозначно продолжается до гомеоморфизма $\Psi$ с требуемым свойством. Предложение доказано.

5.2. Обратно, для поверхности $S \subset \mathbb{P}^{r}$ проекция $f: S \rightarrow \mathbb{P}^{2}$ определяется точкой Грассманиана $\mathrm{Gr}_{r+1, r-2}$ (базисным множеством проекции). Хорошо известно, что множество общих проекций находится во взаимно однозначном соответствии с некоторым открытым по Зарискому подмножеством $U_{S}$ в $\mathrm{Gr}_{r+1, r-2}$. Непрерывная вариация точки в $U_{S}$ задает непрерывное семейство общих проекций поверхности $S$, кривые ветвления которых принадлежат одному и тому же непрерывному семейству плоских каспидальных кривых. Следовательно, дискриминантные кривые двух общих проекций поверхности $S \subset \mathbb{P}^{r}$ принадлежат одной и той же неприводимой компоненте пространства модулей $\mathscr{M}(2 d, g, c)$. 
Более того, если две поверхности $S_{1}$ и $S_{2}$ обшего типа с одинаковыми $K_{S}^{2}=k$ и $p_{a}=p$ вложены с помощью $m$-го канонического класса в одно и то же проективное пространство $\mathbb{P}^{r}$ и принадлежат одной и той же неприводимой компоненте грубого пространства модулей $\mathscr{M}_{S}(k, p)$ поверхностей с заданными инвариантами [12], то существуют общие проекции $f_{1}$ поверхности $S_{1}$ и $f_{2}$ поверхности $S_{2}$, принадлежащие одному и тому же непрерьвному семейству общих проекций. Следовательно, дискриминантные кривые двух обших проекций поверхностей $S_{1}$ и $S_{2}$, принадлежащих одной и той же неприводимой компоненте пространства модулей $\mathscr{M}_{S}(k, p)$, принадлежат одной и той же неприводимой компоненте пространства $\mathscr{M}(2 d, g, c)$ (см. [27]). По теореме 3 и предложениям 5 и 6 для поверхности общего типа с обильным каноническим классом тройка целых чисел $(m, k, p)$ однозначно определяется инвариантами $(d, g, c) \quad m$-канонической дискриминантной кривой; также верно и обратное утверждение. Следовательно, по предложению 1 и теореме 3

$$
i(2 d(k, p), g(k, p), c(k, p)) \geqslant i(k, p),
$$

где $i(2 d, g, c)$ (соответственно $i(k, p))$ - число неприводимых компонент пространства $\mathscr{M}(2 d, g, c)$ (соответственно $\left.\mathscr{M}_{S}(k, p)\right)$.

В [4] $\Phi$. Катанезе показал, что для каждого натурального $h$ существуют такие $k$ и $p$, что $\mathscr{M}_{S}(k, p)$ имеет по крайней мере $h$ неприводимых компонент (подробнее об этом см. в следуюшем параграфе). Следовательно, для каждого натурального $h$ существуют такие $d, g, c$, что $\mathscr{M}(2 d, g, c)$ имеет по крайней мере $h$ неприводимых компонент. Отметим, что оценки снизу для $i(k, p)$, полученные в [4] и [18], также имеют место и для $i(2 d, g, c)$, где $d, g$ и $c$ - инварианты соответствуюших $m$-х канонических дискриминантных кривых.

5.3. Недавно было опубликовано несколько статей (см., например, [1], [22], $[25],[26])$, посвященных парам Зариского. По определению Артал-Бартоло две плоские кривые $C_{1}, C_{2} \subset \mathbb{P}^{2}$ называются парой Зариского, если они одной и той же степени и имеют гомеоморфные трубчатые окрестности в $\mathbb{P}^{2}$, но пары $\left(\mathbb{P}^{2}, C_{1}\right)$ и $\left(\mathbb{P}^{2}, C_{2}\right)$ не гомеоморфны.

Первый пример такой пары был получен О. Зариским, и это - как раз две кривые степени 6 с шестью каспами, упомянутые во введении.

В силу теоремы 3 , применяя предложение 8 , легко доказать существование большого числа различных пар Зариского. Для этого мы можем найти пары негомеоморфных минимальных поверхностей общего типа с обильньм каноническим классом и одинаковыми $K_{S}^{2}$ и $p_{a}$ и рассмотреть соответствуюшие им $m$-канонические дискриминантные кривые. Например, имеем следующее предложение.

ПРЕДЛОЖЕНИЕ 9. Для каждого челого $m \geqslant 5$ существует по крайней мере одна пятерка Зариского (даже не пара) плоских каспидальных кривых $B_{m, i}$, $i=1, \ldots, 5$, степени $m(3 m+1)$ и рода $g=(1 / 2)(3 m+1)(3 m+2)+1 c$ $c=3\left(4 m^{2}+3 m-3\right)$ каспами.

Доказательство. Сушествует (см. [13], [24], [2]) пять негомеоморфных поверхностей $S_{i}(i=1, \ldots, 5)$ общего типа с $p_{g}=0, p_{a}=1$ и $K_{S_{i}}^{2}=1$. Они различаются с помошью Tors $H_{1}\left(S_{i}, \mathbb{Z}\right)=\mathbb{Z} / i \mathbb{Z}$. Согласно [5] для каждого $i, 1 \leqslant i \leqslant 5$, сушествуют поверхности $S_{i}$ с обильным каноническим классом, имеюшие данные 
инварианты. Пусть $\phi_{m, i}: S_{i} \hookrightarrow \mathbb{P}^{m(m-1) / 2}-m$-каноническое вложение, $m \geqslant 5$ (см. [3]), и $f_{m, i}: S_{i} \rightarrow \mathbb{P}^{2}-$ общая проекция с дискриминантной кривой $B_{m, i}$. Применяя вычисления, проведенные в доказательстве теоремы 3 , получаем, что кривая $B_{m, i}$ имеет

$$
\operatorname{deg} B_{m, i}=m(3 m+1), \quad g=\frac{1}{2}(3 m+1)(3 m+2)+1, \quad c=3\left(4 m^{2}+3 m-3\right) .
$$

По предложению 8 , так как $S_{i}$ и $S_{j}$ не гомеоморфны для $i \neq j$, пары $\left(\mathbb{P}^{2}, B_{m, i}\right)$ и $\left(\mathbb{P}^{2}, B_{m, j}\right)$ также не гомеоморфны.

Простейший путь построить две негомеоморфные поверхности $S_{1}$ и $S_{2}$ общего типа, для которых общие морфизмы, заданные линейными системами $m$-канонического класса, приводят к паре Зариского $B_{1}$ и $B_{2}$, - это построить поверхности с разными иррегулярностями $q_{1}$ и $q_{2}$, где $q_{i}=\operatorname{dim} H^{1}\left(S_{i}, \mathscr{O}_{S_{i}}\right)$, и с одинаковыми $K_{S}^{2}$ и $p_{a}$. Например, пусть $A$ - абелева поверхность и $C \subset A$ - такая неособая кривая, что класс кривой $C$ в $\mathrm{Pic} A$ делится на 2. Рассмотрим двулистное накрытие $\varphi$ : $S \rightarrow A$, разветвленное вдоль $C$. Легко проверить, что $S$ - поверхность общего типа с обильным каноническим классом и иррегулярностью $q \geqslant 2$. Если $C^{2}=8 p$, то простейшие вычисления дают $K_{S}^{2}=4 p$ и $p_{a}=p$. С другой стороны, У. Перссон доказал в [23], что для любых натуральных $x, y$, удовлетворяюших неравенствам

$$
2 x-6 \leqslant y \leqslant 8\left(x-c x^{\frac{2}{3}}\right),
$$

где $c=9 / \sqrt[3]{12}$, существует односвязная минимальная поверхность общего типа с $K^{2}=y$ и $p_{a}=x$. Легко видеть, что $x=p$ и $y=4 p$ удовлетворяют $(41)$, если $p \geqslant 486$.

\section{§6. Доказательство теоремы 13}

6.1. В [4] и [5] Катанезе исследовал простое бидвойное накрытие $\varphi: S \rightarrow Q=$ $\mathbb{P}^{1} \times \mathbb{P}^{1}$ битипа $(a, b),(m, n)$, т.е. $\varphi$ - конечное $(\mathbb{Z} / 2 \mathbb{Z})^{2}$ накрытие Галуа квадрики $\mathbb{P}^{1} \times \mathbb{P}^{1}$, разветвленное вдоль двух обших кривых соответственно бистепеней $(2 a, 2 b)$ и $(2 m, 2 n)$. Пусть $a, b, m, n$ - целые числа, удовлетворяющие следующим условиям:

$$
\begin{aligned}
& a>2 n, \quad n \geqslant 3, \quad m>2 b, \quad b \geqslant 3, \\
& a \equiv n(\bmod 2), \quad b \equiv m(\bmod 2) .
\end{aligned}
$$

Положим

$$
u=(n+a-2), \quad v=(m+b-2), \quad w=(a-n), \quad z=(m-b) .
$$

Согласно [4] для бидвойного накрытия $\varphi: S \rightarrow Q=\mathbb{P}^{1} \times \mathbb{P}^{1}$ битипа $(a, b),(m, n)$ поверхность $S$ является односвязной поверхностью общего типа с каноническим классом

$$
K_{S}=\varphi^{*}\left(\mathscr{O}_{Q}(u, v)\right)
$$


и

$$
K_{S}^{2}=8 u v, \quad \chi=\chi\left(\mathscr{O}_{S}\right)=\frac{3}{2} u v+(u+v)+2-\frac{1}{2} w z,
$$

где $\chi$ - Эйлерова характеристика структурного пучка $\mathscr{O}_{S}$ на $S$.

Для $u, v, w, z$, удовлетворяющим (42), канонический класс $K_{S}$ является 2-делимым в $H^{2}(S, \mathbb{Z})$, а индекс

$$
r=r(S)=\max \left\{s \in \mathbb{N} \mid\left(\frac{1}{s}\right) K_{S} \in H^{2}(S, \mathbb{Z})\right\}
$$

равен наибольшему общему делителю $(u, v)$.

Так как $K_{S}$ является 2-делимым, то форма пересечения на $H_{2}(S, \mathbb{Z})$ является четной. Кроме того, по теореме Фридмана [11] два таких бидвойных накрытия $S_{1}$ и $S_{2}$ квадрики $Q$ гомеоморфны тогда и только тогда, когда $K_{S_{1}}^{2}=K_{S_{2}}^{2}$ и $\chi\left(\mathscr{O}_{S_{1}}\right)=$ $\chi\left(\mathscr{O}_{S_{2}}\right)$. В [4] и [5] было доказано, что для каждого целого $k$ существует по крайней мере один набор $\left(S_{1}, \ldots, S_{k}\right)$, состоящий из $k$ бидвойных накрытий квадрики $Q$ битипов $\left(a_{i}, b_{i}\right),\left(m_{i}, n_{i}\right)$, удовлетворяющий описанным выше условиям и такой, что:

(i) $S_{i}$ и $S_{j}$ гомеоморфны друг другу при $1 \leqslant i, j \leqslant k$;

(ii) $r\left(S_{i}\right) \neq r\left(S_{j}\right)$ при $i \neq j$ (и, следовательно, $S_{i}$ и $S_{j}$ не диффеоморфны).

Мы будем называть наборы поверхностей общего типа, удовлетворяющие условиям (i) и (ii), наборами Катанезе.

ПримеР. Два бидвойных накрытия $S_{1}$ битипа $(16,22),(52,4)$ и $S_{2}$ битипа $(28,10),(28,10)$ являются парой Катанезе. Из формул $(1)-(3)$ следует, что эти поверхности имеют

$$
\begin{array}{cc}
K_{S}^{2}=K_{S_{i}}^{2}=10368, & \chi=\chi\left(\mathscr{O}_{S_{i}}\right)=1456, \\
r\left(S_{1}\right)=18, & r\left(S_{2}\right)=36 .
\end{array}
$$

Рассмотрим $m$-каноническое вложение $\Phi_{\left|m K_{S_{i}}\right|}: S_{i} \rightarrow \mathbb{P}^{r}, m \geqslant 5$ (см. [3]), и пусть $f_{m, i}: S_{i} \rightarrow \mathbb{P}^{2}$ - ограничение на $\Phi_{\mid m K_{S_{i} \mid}}\left(S_{i}\right)$ общей проекции pr: $\mathbb{P}^{r} \rightarrow \mathbb{P}^{2}$. Обозначим через $R_{m, i} \subset S_{i}$ кривую ветвления общей проекции $f_{m, i}$ и через $B_{m, i} \subset \mathbb{P}^{2}$ дискриминантную кривую морфизма $f_{m, i}$.

Пусть общий морфизм $f_{m}: S \rightarrow \mathbb{P}^{2}$ задан трехмерной линейной подсистемой $m$-канонического класса на $S$. Тогда из формулы присоединения

$$
K_{S}=f^{*}\left(K_{\mathbb{P}^{2}}\right)+R_{m}
$$

следует, что кривая ветвления $R_{m} \subset S$ морфизма $f_{m}$ принадлежит $\left|(3 m+1) K_{S}\right|$. Следовательно, если мы имеем два обших морфизма $f_{i, m}: S_{i} \rightarrow \mathbb{P}^{2}$, заданных трехмерными линейными подсистемами из $m$-канонических классов на $S_{i}$ и таких, что соответствуюшие пары $\left(\mathbb{P}^{2}, B_{1, m}\right)$ и $\left(\mathbb{P}^{2}, B_{2, m}\right)$ гомеоморфиы, то по предложению 8 (согласно теореме 3 гипотеза Кизини выполнена для $B_{1}$ ) существует гомеоморфизм $\Psi: S_{1} \rightarrow S_{2}$ такой, что $\Psi\left(R_{1, m}\right)=R_{2, m}$. В этом случае должно выполняться равенство $r\left(S_{1}\right)=r\left(S_{2}\right)$, так как $\Psi$ индуцирует изоморфизм $\Psi_{*}: H_{2}\left(S_{1}, \mathbb{Z}\right) \rightarrow$ $H_{2}\left(S_{2}, \mathbb{Z}\right)$, для которого выполнено условие $\Psi_{*}\left(R_{1, m}\right)=R_{2, m}$. Но это невозможно, так как по определению $r\left(S_{1}\right) \neq r\left(S_{2}\right)$ для пары Катанезе $S_{1}, S_{2}$. Теорема 13 доказана. 
ПримеР (продолжение). Применяя теорему 1 для $m$-канонических дискриминантных кривых обших морфизмов поверхностей $S_{1}$ и $S_{2}$ в рассмотренном вьше примере, мы получим пары Зариского $\left(B_{1, m}, B_{2, m}\right), m \geqslant 5$, плоских каспидальных кривых. Применяя вычисления из доказательства теоремы 3 , легко видеть, что $m$-канонические дискриминантные кривые $B_{i, m}$ имеют степень $\operatorname{deg} B_{i, m}=$ $10368 m(3 m+1)$, геометрический род $g=5184(3 m+2)(3 m+1)+1$ и $c=$ $10368\left(12 m^{2}+9 m\right)-13632$ обыкновенных каспов.

\section{Список литературы}

1. Artal-Bartolo E. Sur les couples de Zariski // J. Alg. Geom. 1994. V. 3. № 2. P. 223-247.

2. Barlow R. Some new surfaces with $p_{g}=0$ // Duke Math. J. 1984. V. 51. № 4. P. 889-904.

3. Bombieri E. Canonical models of surfaces of general type // Publ. Math. IHES. 1973. V. 42. P. 171-219.

4. Catanese F. On the moduli space of surfaces of general type // J. Diff. Geom. 1984. V. 19. P. 483-515.

5. Catanese F. Connected components of moduli space // J. Diff. Geom. 1986. V. 24. P. 395-399.

6. Catanese F. On a Problem of Chisini // Duke Math. J. 1986. V. 53. № 1. P. 33-42.

7. Catanese F., Le Brun C. On the scalar curvature of Einstein manifolds // Math. Research Letters. 1997. V. 4. № 6.

8. Catanese F., Ciliberto C., Mended Lopes M. On the classification of irregular surfaces of general type with nonbirational bicanonical map // Transactions of AMS. 1988. V. 350. № 1.

9. Chisini $O$. Sulla identita birazionale delle funzioni algebriche di due variabili dotate di una medesima curva di diramazione // Rend. Ins. Lombardo. 1944. V. 77. P. 339-356.

10. Ein L., Lazarsfeld $R$. Syzygies and Koszul cohomology of smooth projective varieties of arbitrary dimension // Invent. Math. 1993. V. 111. P. 51-67.

11. Freedman M. H. The topology of four dimensional manifolds // J. Diff. Geom. 1982. V. 17. P. 357-453.

12. Gieseker D. Global moduli for surfaces of general type // Invent. Math. 1977. V. 43. P. 233-282.

13. Godeaux L. Sur une surface algebrique de genre zero et de bigenre deux // Atti Accad. Naz. Lincei. 1931. V. 14. P. 479-481.

14. Kulikov Vik.S. On the fundamental group of the complement of a hypersurface in $\mathbb{C}^{n}$ // Springer L.N.M. 1991. V. 1479. P. 122-130.

15. Куликов Вик. С. Фундаментальная группа дополнения к гиперповерхности в $\mathbb{C}^{n} / /$ Изв. АН CССР. Сер. матем. 1991. Т. 55. № 3. С. 434-455.

16. Куликов Вик. C. Геометрическая реализация $C$-групп // Изв. РАН. Сер. матем. 1994. T. 58. №4. C. 194-203.

17. Куликов Вик. С. О структуре фундаментальной группы дополнения к алгебраической кривой $\mathbb{C}^{2}$ // Изв. РАН. Сер. матем. 1992. Т. 56. № 2. С. 469-480.

18. Manetti $M$. Degeneration of algebraic surfaces and applications to moduli problem: Tesi di perfezionamento. Scuola Normale Superiore. Pisa, 1996.

19. Moishezon B. Stable branch curves and braid monodromies // Springer L.N.M. 1981. V. 862. P. 107-192.

20. Moishezon B., Teicher M. Braid group technique in complex geometry. II: from arrangements of lines and conics to cuspidal curves // Springer L.N.M. 1991. V. 1479. P. 131-180.

21. Nori M. Zariski's conjecture and related problems // Ann. Sci. École Norm. Sup. Ser. 4. 1983. V. 16. P. 305-344.

22. Oka M. Two transforms of plane curves and their fundamental groups // J. Math. Sci. Univ. Tokyo. 1996. V. 3. P. 399-443. 
23. Persson U. Chern invariants of surfaces of general type // Compos. Math. 1981. V. 43. № 1. P. 3-58.

24. Reid $M$. Surfaces with $p_{g}=0, K^{2}=1 / /$ J. Fac. Sci. Univ. Tokyo Sect. IA Math. 1978 . V. 25. № 1. P. 75-92.

25. Shimada I. A note on Zariski pairs // Compos. Math. 1996. V. 104. № 2. P. 125-133.

26. Tokunaga H. A remark on E. Artal-Bartolo's paper: "On Zariski pairs" // Kodai Math. J. 1996. V. 19. P. 207-217.

27. Wahl J. Deformations of plane curves with nodes and cusps // Amer. J. Math. 1974. V. 96. P. 529-577.

28. Zariski $O$. On the topological discriminant group of a Riemann surface of genus $p$ // Amer. J. Math. 1937. V. 59. P. 335-358.

29. Zariski O. Algebraic surfaces. Berlin-N.Y.: Springer-Verlag, 1971.

Математический институт им. В. А. Стеклова РАН

E-mail: victor@olya.ips.ras.ru
Поступило в редакцию 26.V.1998

22.IX.1998 